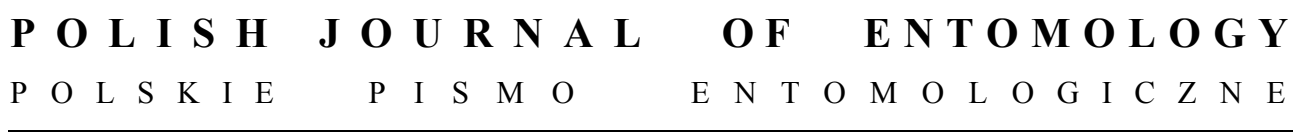

VOL. 80: $83-116$

Gdynia

31 March 2011

DOI: $10.2478 / \mathrm{v} 10200-011-0007-2$

\title{
Contribution to knowledge of the butterflies and moths (Lepidoptera) of north-eastern Poland with a description of a new tineid species from the genus Monopis HüBNER, 1825
}

\author{
JAN ŠUMPICH *, JAN LIŠKA **, IVO DVOŘÁK ***
}

* CZ-582 61 Česká Bělá 212, Czech Republic; e-mail: jansumpich@seznam.cz;

** Forestry and Game Management Research Institute Jíloviště-Strnady, CZ-156 04

Prague 5 - Zbraslav, Czech Republic; e-mail: liska@vulhm.cz;

*** Tylova 23, CZ-58601 Jihlava, Czech Republic; e-mail: ivo.dvorak@post.cz

\begin{abstract}
This work contains faunistic data on the occurrence of 677 butterfly and moth species found during 2000-2008 in north-eastern Poland (Podlasie Province). The species Monopis fenestratella (HEYDEN, 1863), Amphisbatis elsae SVENSSON, 1982, Coleophora ptarmicia WALSINGHAM, 1910 and Epermenia falciformis (HAWORTH, 1828) were found in Poland for the first time. Recent data are provided for five other species - Monochroa servella (ZELLER, 1839), Teleiodes aenigma SATTLER, 1983, Dichomeris limosella (SCHLÄGER, 1849), Aethes rutilana (HÜBNER, 1817) and Eana derivana (LA HARPE, 1858) - known in Poland only from historical data. The occurrence in Podlasie of 75 species is reported for the first time, and the occurrence of 6 other species is confirmed for this area after more than 50 years. This work also describes a new species, Monopis bisonella ŠUMPICH, sp. n. A number of species are very rare in Poland and occur only locally. These include Nemapogon wolffiellus KARSHOLT \& NIELSEN, 1976, Coleophora trifariella ZELlER, 1849, Sophronia chilonella (TREITSChKE, 1833), Syncopacma sangiella (StAINTON, 1863), Helcystogramma albinerve (GERASIMOV, 1929), Cochylis flaviciliana (WeStwoOd, 1854), Acleris comariana (LIENIG \& ZELLER, 1846), Apotomis semifasciana (HAWORTH, 1811), Cydia coniferana (SAXESEN, 1840), Euphydryas maturna (LinNAEUS, 1758), Lopinga achine (SCOPOLI, 1763), Scopula nemoraria (HÜBNER, 1799), Epirrhoe tartuensis MOELS, 1965 and Schrankia taenialis (HÜBNER, 1809). A complete list of the species found is provided in the Appendix at the end of this work.
\end{abstract}

KEY WORDS: Lepidoptera, faunistics, new records, Monopis bisonella sp. n., Podlasie, Poland.

\section{INTRODUCTION}

This report summarizes findings about the lepidopteran fauna of north-eastern Poland 
obtained during several random excursions in 2000-2008. The first collections were made in 2000 in association with the international meeting of lepidopterologists in Białowieża held by Societas Europaea Lepidopterologica (SEL). The field excursions of J. Šumpich and I. Dvořák took place in the close vicinity of Białowieża at that time, and the findings were recorded in written form. In 2006, the same authors took part in the 24th meeting of Polish lepidopterologists in the Biebrza National Park, where they examined material near the village of Osowiec Twierdza during very bad weather. The fundamental results presented in this report, however, were obtained by J. Šumpich and J. Liška during their third visit to north-eastern Poland in 2008, namely, to the region around the town of Augustów. During five night-time excursions in the neighbourhoods of Kukowo and Płaska several hundred species were found, a number of which turned out to be faunistically interesting not only for Poland but also in a broader central European context.

Most of the species found were caught using a $125 \mathrm{~W}$ mercury lamp and an $8 \mathrm{~W}$ fluorescent lamp. Additional adults were caught with a lepidopterological net (sweeping, by individual catching). The material evidence is filed in the authors' collections.

\section{Acknowledgement}

We would like to thank prof. dr J. Buszko for reviewing the manuscript. We also thank K. Šmerdová and A. Kirking for correcting the English. And finally we would like to thank mgr. inż. A. Rodziewicz (Kukowo) for kindly invitation, accompanyment and accomodation in the year 2008 .

\section{SYSTEMATICS}

\section{Monopis fenestratella (HEYDEN, 1863)}

(Tineidae)

\section{Material examined}

Płaska, 1 female (Fig. 7), 17 VI 2008 (leg., coll. J. Šumpich). The specimen was captured at light in open woodland.

The species is known from all the countries surrounding Poland with the exception of Belarus (GAEDIKE, in KARSHOLT \& NIEUKERKEN 2004); it is very rare in central Europe, as evidenced by the very small number of records from these neighbouring countries. In Slovakia, it was recorded for the first time at Prievidza in 1998 (TOKÁR et al. 2002). In the Czech Republic, it was found only in northern and southern Moravia in 2007 (SITEK 2008). We also know of a very few findings from Germany and Ukraine (PETERSEN 1969, PETERSEN \& GAEDIKE 1987). Apart from central Europe, the species occurs in northern and north-western Europe including the British Isles. For Poland, it is a new species. 


\section{Monopis bisonella S̆UMPICH, sp. $\mathbf{n}$.}

(Tineidae)

\section{Material examined}

Holotype: male, Białowieża env., Podolany village, (genitalia in glycerine in the plastic tube on the pins below the adult) (Fig. 1), 28 V 2000 (leg., coll. J. Šumpich, later in Czech museum with author's collection).

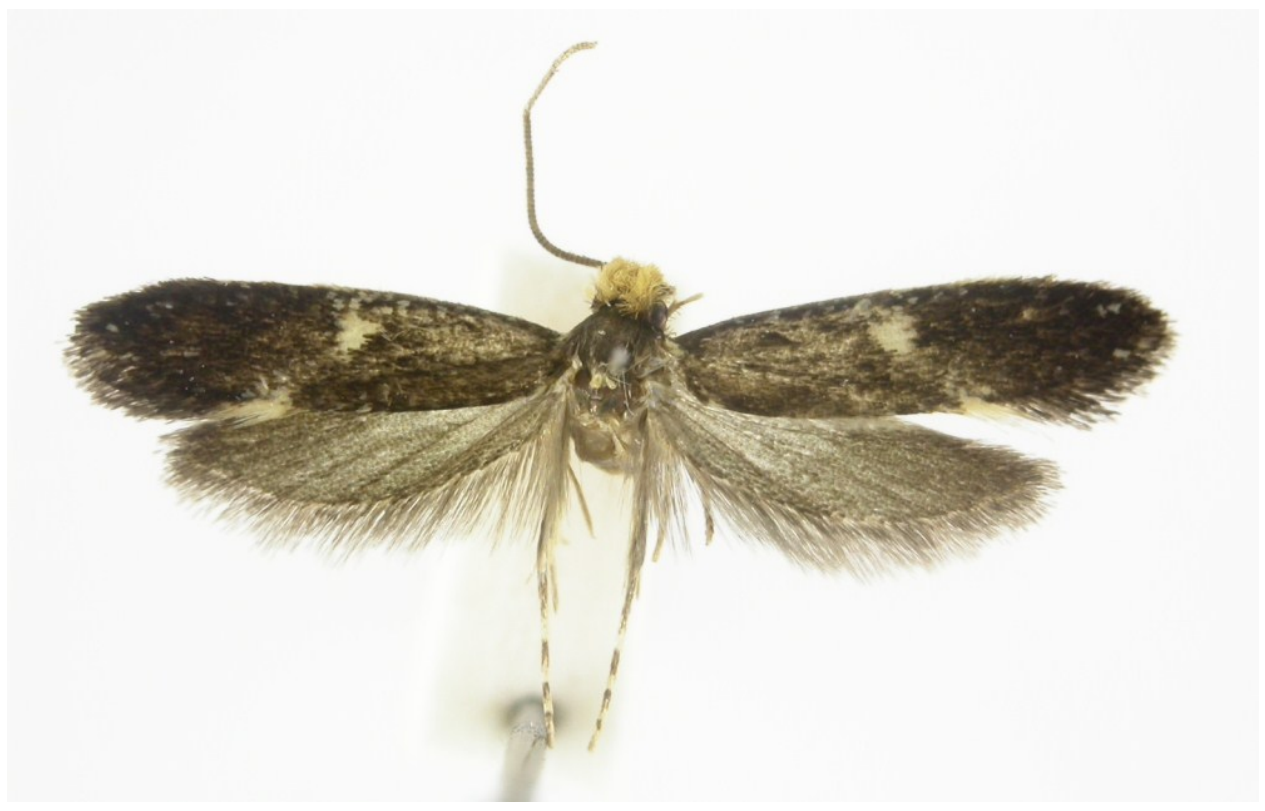

Fig. 1. Holotype of Monopis bisonella sp. n.

\section{Description of male}

Wingspan: $11 \mathrm{~mm}$. Head and frons densely covered with yellow ochre bristles (hairs). Labial palps yellow when fully extended. Antenna filiform, dark, the last third white. Thorax and abdomen brown, concolorous with the forewings. Leg undersides yellow, upper sides alternately black and yellow. Forewing: ground colour (Fig. 1) dark brown; in the centre, a bold, sharply defined white spot. A second white spot on the dorsum, the white colour extending to the fringes. Hindwings brown, paler than forewings. Fringes of both wings the same hue as hindwing except where the white spot meets the forewing edge.

Male genitalia (Figs 2-4): uncus oblong, bottle-shaped, tapering towards the tip; tip bifurcate with pointed terminations. Gnathos tapering gradually, convex in the middle, bent, with a pointed termination. A spur protrudes just below the termination. Valva elongate, oval, $2.5 \mathrm{x}$ longer than its width in the middle; at the base it is wider than at the outer end. 
Ventral border gently curved, dorsal side is very much convex; the shape of the valve remotely resembles the letter S. Saccus 1.2 x longer than aedeagus, very narrow, tapering slightly in bottom fifth of its length. Aedeagus narrow, $2.5 \mathrm{x}$ wider than saccus, slightly curved in the lateral aspect, $1.5 \mathrm{x}$ longer than valva, without cornutus. Vesica densely covered with small striae.

Female: unknown.

Biology: unknown. The holotype was caught on a wet meadow close to a wet forest. Presumably, the ecological requirements of this species are similar to those of most species of the genus Monopis.

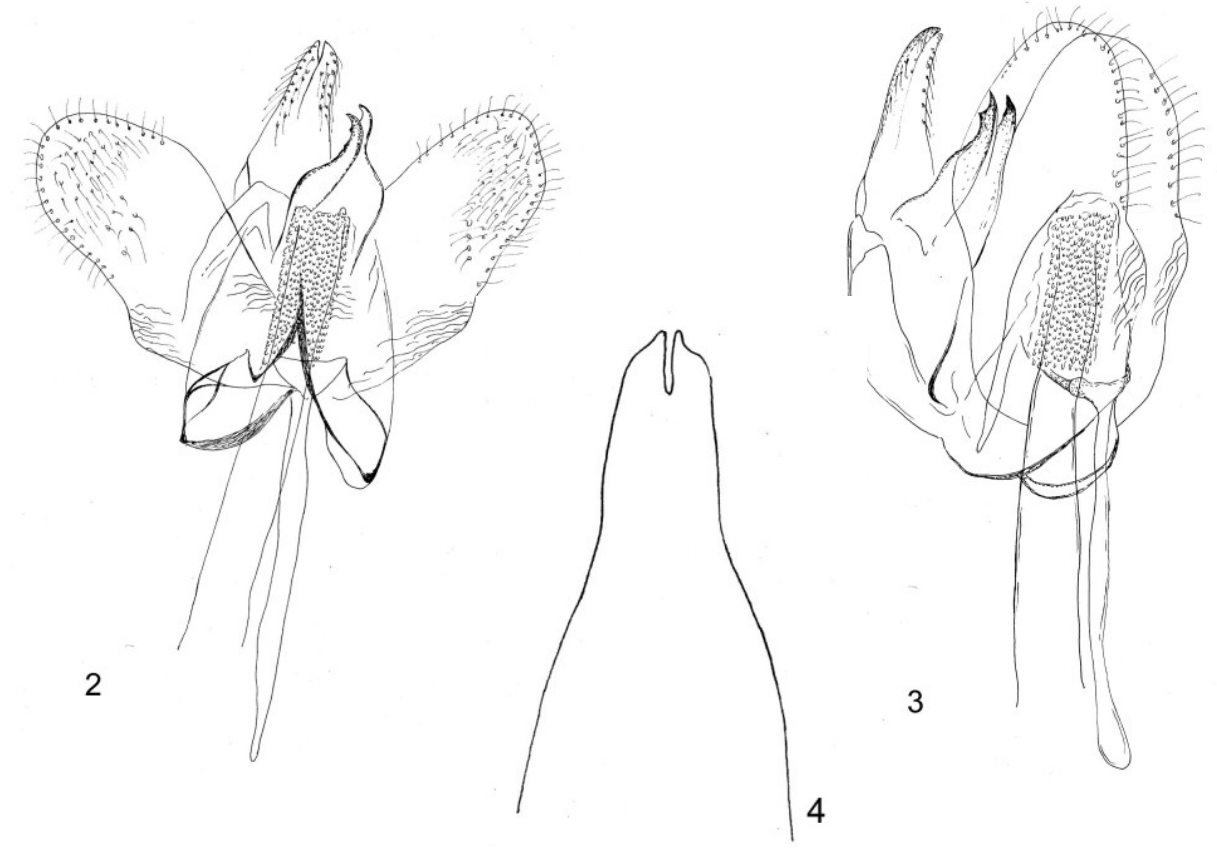

Figs 2-4. Male genitalia of Monopis bisonella sp. n., 2 - ventral aspect, 3 - lateral aspect, 4 - ventral aspect of uncus.

\section{Distribution}

The type locality is in north-eastern Poland (Fig. 5), which is the south-western border of the European boreal zone. Therefore, it may well be of boreal origin and has spread into a wider area. 


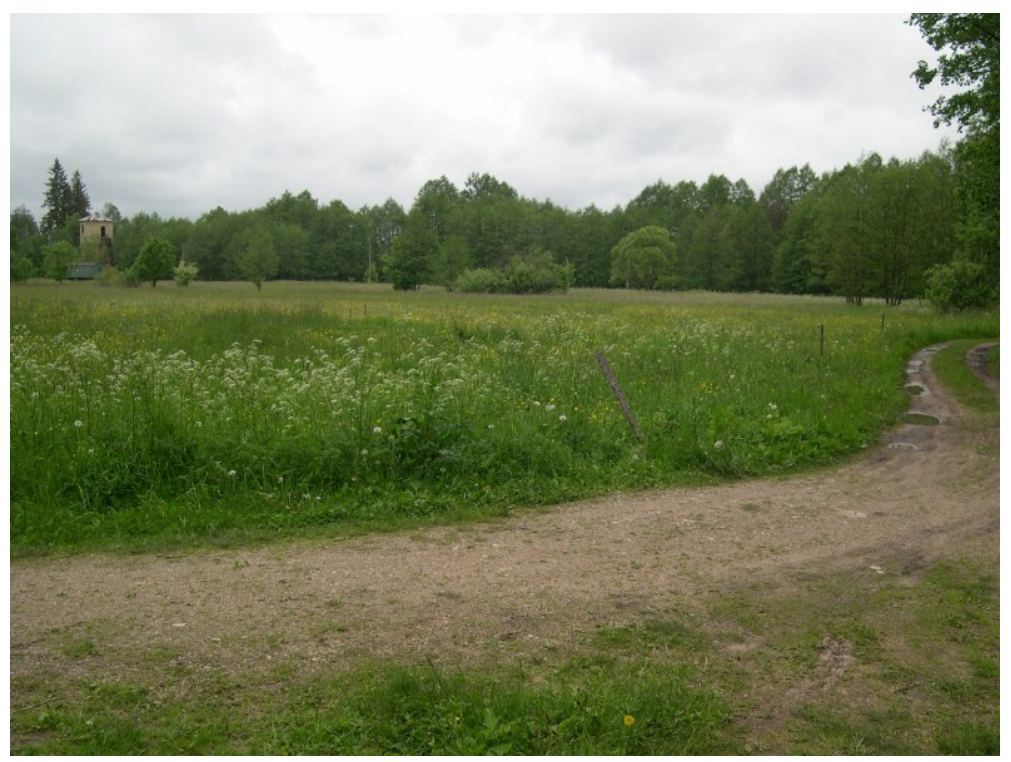

Fig. 5. The habitat of Monopis bisonella sp. n. and the other species presented in this paper (Podolany near Białowieża).

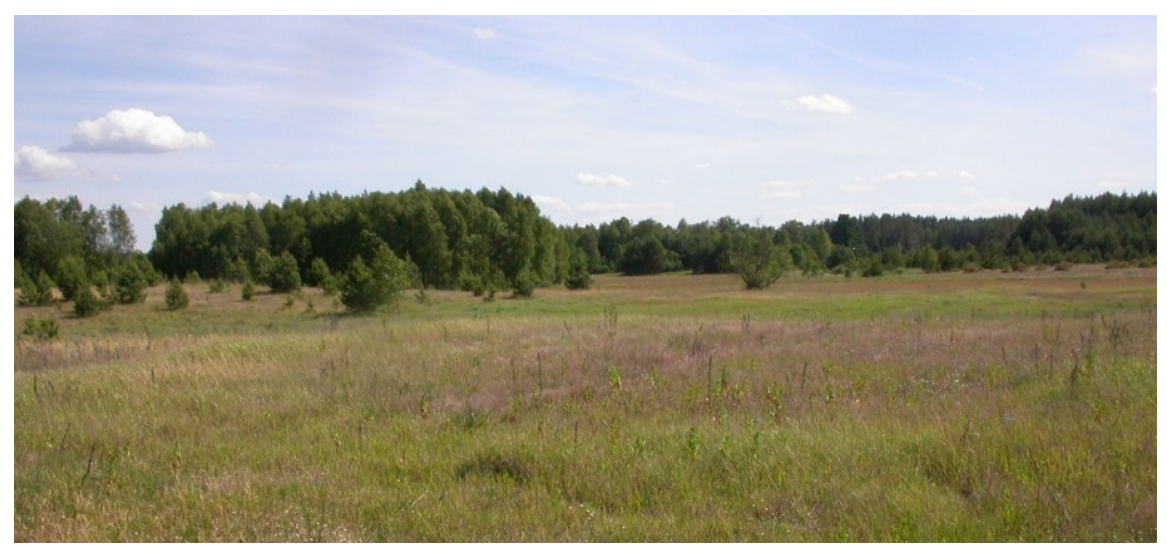

Fig. 6. The habitat of the most interesting species presented: Płaska.

\section{Etymology}

The name of the new species is derived from the generic name of the European bison (Bison bonasus), as the population of these mammals is numerous precisely where the newly described moth was found.

\section{Remarks}

Nowadays, the genus Monopis HÜBNER, 1825 includes 135 species (ROBINSON 2002), 
just 14 of which occur in Europe (GAEDIKE, in KARSHOlt \& NIEUKERKEN 2004). Monopis bisonella ŠUMPICH, sp. n. can be relatively easily distinguished from other similar Palaearctic species of this genus (Petersen 1957, ZAGUlJaev 1960, GaediKe \& KARSHOLT 2001, XIAO \& LI 2006), thanks to its two sharply defined spots on the forewing (the most similar species by size, M. fenestratella, has one spot with a fuzzy border). Compared to the species in the M. laevigatella group, which have a very similar forewing pattern, Monopis bisonella ŠUMPICH, sp. $\mathrm{n}$. is significantly smaller and differs from most of the former species in the chocolate colour of its forewings. We can reliably differentiate the species by the male genitalia: the combination of the characteristically shaped gnathos and convex valve is a definitive characteristic.

\section{Parornix avellanella (STAINTON, 1854)}

(Gracillariidae)

\section{Material examined}

Kukowo, 2 males, 17 VI 2008 (leg., coll. J. Šumpich). A widespread species, the name of which was until recently considered to be a synonym of $P$. devoniella (STAINTON, 1850) (PRINS \& PRINS 2005). The material found in north-eastern Poland was identified according to KUZNETZOV \& BARYSCHNIKOVA (2003), who revised the taxonomic position of the Palaearctic species of the genus Parornix SPULER, 1910.

\section{Scythris palustris (ZELLER, 1855)}

(Scythrididae)

\section{Material examined}

Białowieża env., Podolany village, 1 male, 28 V 2000 (leg., coll. J. Šumpich). A wetland species, its exclusive foodplant is Rhytidiadelphus squarrosus (BENGTSSON 1997). The range of distribution of this species is concentrated in central Europe, but it has also been known from western and north-eastern Europe (BENGTSSON in KARSHOLT \& NIEUKERKEN 2004). In Poland it is very local. It used to be found in the provinces of Western Pomerania, Lower Silesia and Małopolska, but recently just in Lublin Province (BUSZKO \& NOWACKI 2000).

\section{Coleophora ptarmicia WALSINGHAM, 1910}

(Coleophoridae)

\section{Material examined}

Płaska, 1 male (Fig. 8), 17 VI 2008 (leg., coll. J. Šumpich). The specimen was caught at light in an open, steppe-like biotope with sandy soil. The caterpillar is associated with Achillea millefolium, which occurs in this locality in great abundance. A very local species; to date, there is insufficient knowledge regarding its occurrence. Today, the species is known from just a few countries in central and western Europe, the Baltic countries and Russia (BALDIZZONE \& van der Wolf in KARSHOlt \& NiEUKERKEN 2004). Among 
Poland's neighbouring countries, it has been found only in the Czech Republic (STERNECK \& ZiMMERMANN 1933, GREGOR et al. 1984, ŠUMPICH et al. 2007) and in Slovakia (REIPRICH \& OKÁLI 1989). A new species for Poland.

\section{Pseudatemelia elsae SVENSSON, 1982}

\section{Material examined}

(Amphisbatidae)

Płaska, 25 spec. (not sexed) (Fig. 9), 17 VI 2008 (leg., coll. J. Šumpich, J. Liška), many others were sighted. Material evidence was obtained by catching at light at the edge of a pine forest. We do not have sufficiently reliable information regarding the occurrence of this species, as it is very similar to related species of this genus and can very easily be confused with them. Current knowledge of the life history, habitats and distribution is summarized by TOKÁR et al. (2005). In neighbouring countries only known from the Czech Republic (LIŠKA et al. 2001) and Slovakia (TOKÁR et al. 2002). A new species for Poland.

\section{Monochroa servella (ZELLER, 1839)}

(Gelechiidae)

\section{Material examined}

Płaska, 1 male, 17 VI 2008 (leg., coll. J. Liška). The specimen was caught individually in a wetland biotope near the lake "Jezioro Pobojno". A very local species (and very rare in most of the locations where it occurs), its food plants are Primula farinosa and P. veris (ELSNER et al. 1999). The species occurs in pockets across the whole of Europe, including all of Poland's neighbours (KARShOlt in KarShOLT \& NieUKERKEN 2004). In Poland to date, there exists only historical data on its occurrence, namely from Western Pomerania and Lower Silesia (BUSZKO \& NOWACKI 2000). The rediscovery of this species in Augustów Forest confirms the occurrence of this species in Poland after a gap of more than 50 years.

\section{Monochroa rumicetella (HOFMANN, 1868)}

(Gelechiidae)

\section{Material examined}

Biebrza National Park, Osowiec Twierdza env., 2 males (Fig. 10), 3 VI 2006 (leg., coll. J. Šmpich). The material was caught at UV light in a dry pine wood with numerous Juniperus communis bushes. The caterpillar is associated with species of Acetosa and Acetosella; it prefers forest-steppe biotopes (ELSNER et al. 1999). Although this species inhabits the whole of Europe, it is very local and its populations are not dense anywhere. In Poland, it has so far been known only from the province of Kujawy-Pomerania, and 
historically Małopolska. The finding in Podlasie makes a significant addition to our knowledge of this species in Poland.

\section{Monochroa niphognatha (GOZMÁNY, 1953)}

\section{Material examined}

(Gelechiidae)

Białowieża env., Podolany village, 1 male (Fig. 11), 28 V 2000 (leg., coll. J. Šumpich). The voucher specimen was caught at light in a meadow, which tallies with present knowledge of this species' ecological requirements (ELSNER et al. 1999). To date, the species has been known from just a few localities in central and northern Europe, and recently it was found in the British Isles and in Ukraine (ELSNER et al. 1999, KARSHOLT in KARShOlt \& NieUKERKEN 2004, BidZILYA \& BUdAShKIN 2009). In Poland, it has so far been recorded in just two provinces (Lublin and Podlasie).

\section{Carpatolechia aenigma SATTLER, 1983}

(Gelechiidae)

\section{Material examined}

Płaska, 1 male (Fig. 12), 17 VI 2008 (leg., coll. J. Šumpich). The voucher specimen was caught at light in an open, steppe-like habitat, which corresponds to the habitat preferences of the species (ELSNER et al. 1999). It occurs mostly in eastern, western and central Europe. It does not occur on the islands of Europe, in the Iberian Peninsula or in the Nordic countries (KARShOlT in KARShOlt \& NieUKerKen 2004). In Poland to date it has been known only from historical data from the province of Mazovia (BUSZKO \& NOWACKI 2000). The present finding from Augustów Forest provides important confirmation of its occurrence in Poland.

\section{Dichomeris limosella (SCHLÄGER, 1849)}

\section{(Gelechiidae)}

\section{Material examined}

Białowieża env., Podolany village, 1 specimen, 21 VI 2008 (observ. J. Šumpich, J. Liška). The present specimen was observed on a forest path, where it was attracted to light. This species is widespread in Europe (KARSHOLT in KARSHOLT \& NIEUKERKEN 2004), and it is not so rare in a number of places (hence, no voucher specimens were collected). However, only historical records are known from Poland (BUSZKO \& NOWACKI 2000) and Bohemia, western part of the Czech Republic (NovÁK et al. 1997). This is the first confirmed occurrence in Poland for more than 50 years. 
Aethes rutilana (HÜBNER, 1817)

(Tortricidae)

\section{Material examined}

Płaska, 2 males, 17 VI 2008 (leg., coll. J. Šumpich). Both voucher specimens were attracted to light at the edge of a forest where Juniperus communis is common, with which the caterpillar is uniquely associated. This species is widespread in Europe. Among neighbouring countries, it has not yet been reported in Ukraine, Belarus or Russia (AARVIK in KARSholt \& NieUKerken 2004). In Poland, this species has been found in most provinces but there are no recent data (BUSZKO \& NOWACKI 2000). The situation is similar in the Czech Republic, where the species was mentioned by a number of authors in the first half of the 20th century (STERNECK \& ZIMMERMANN 1933, VLACH 1936, ZIMMERMANN \& SKALA 1947), but recently there has been just one record (ŠUMPICH et al. 2007). The present finding confirms the species' occurrence in Poland after more than 50 years.

\section{Cochylidia moguntiana (RöSSLER, 1864)}

(Tortricidae)

\section{Material examined}

Płaska, 14 males (Fig. 13), 2 females, 16 VI 2008 (leg., coll. J. Šumpich, J. Liška); several others were sighted. The moths were captured by sweeping in dry grasslands and by driving them out from clumps of Artemisia campestris, their food plant. The species occurs in pockets throughout most of Europe but is absent from a number of countries, including Ukraine, Belarus and Russia (AARVIK in KARSHOLT \& NIEUKERKEN 2004); it has not been found in the Czech Republic either (LAŠTU゚VKA \& LIŠKA 2005). In Poland, it is known at present only from Kujawy-Pomerania, but in the past it was also recorded in WarmiaMazury (BUSZKO \& NOWACKI 2000). Its common occurrence in Augustów Forest illustrates the still insufficient knowledge of the moths of north-eastern Poland and highlights the exceptional natural conditions of this region.

\section{Eana derivana (LA HARPE, 1858)}

(Tortricidae)

\section{Material examined}

Płaska, 2 males (Fig. 14), 17 VI 2008 (leg., coll. J. Šumpich, J. Liška). The material was obtained by catching at light on sandy biotopes near the village of Płaska. It is a species whose life history and habitats are generally still unknown, and whose distribution is insufficiently understood. It is known to occur in many European countries but has not been found in the Iberian Peninsula, in the British Isles, or, to date, east of Poland (AARVIK in KARSHOLT \& NIEUKERKEN 2004). From Poland, reports of its occurrence have been published only from the provinces of Pomerania, Silesia and Małopolska, but in all cases 
these are historical data (BUSZKO \& NOWACKI 2000). The present findings confirm the species' recent occurrence in Poland after more than 50 years.

\title{
Cnephasia pasiuana (HÜBNER, 1799)
}

\section{Material examined}

\author{
(Tortricidae)
}

Płaska, 1 male (Fig. 15), 17 VI 2008 (leg., coll. J. Šumpich). The specimen was attracted to a source of ultraviolet light on a forest path. The species is widespread in Europe, its occurrence being reported in most countries (AARVIK in KARSHOLT \& NIEUKERKEN 2004). The species has been found in a number of provinces in Poland, but recently only in Kujawy-Pomerania (BUSZKO \& NOWACKI 2000). The finding contributes to our knowledge of the species' occurrence in Poland, and it provides the first record for Podlasie.

\section{Ancylis diminutana (HAWORTH, 1811)}

(Tortricidae)

\section{Material examined}

Biebrza National Park, Osowiec Twierdza env., 1 male, 3 VI 2006, Kukowo, 1 male, 17 VI 2008 (leg., coll. J. Šumpich). Both specimens were captured at light in wetland habitats. The species is distributed in Europe locally and is come across very infrequently; it is absent from southern Europe and in most countries of western Europe. Among Poland's neighbours, it has been found only in the Czech Republic and in Lithuania (AARVIK in KARSHOLT \& NIEUKERKEN 2004). For Poland, until recently its occurrence was known only from historical records (BUSZKO \& NOWACKI 2000). It was recently reported by RAZOWSKI (2003) from the Borecka Forest (J. Buszko leg.).

\section{Dichrorampha incognitana (KREMKY \& MASLOWSKI, 1933)}

\section{(Tortricidae)}

\section{Material examined}

Płaska, 2 males (Fig. 16), 17 VI 2008 (leg., coll. J. Šumpich). A steppe species associated with Achillea millefolium, on which the material evidence was collected. The species occurs in almost the entire Palaearctic, but it is a very rare species in Europe and is wholly absent from the west and south of the continent (RAZOWSKI 2003, AARVIK in KARSHOLT \& NIEUKERKEN 2004). In Poland, its occurrence is known from very old data from most provinces. It has recently been found only in Kujawy-Pomerania. This is a rare finding, not only from the viewpoint of Polish entomofaunistics but also from the wider European perspective. 


\section{Dichrorampha agilana (TENGSTRÖM, 1848)}

(Tortricidae)

\section{Material examined}

Płaska, 20 spec. (not sexed) (Fig. 17), 16-18 VI 2008 (leg., coll. J. Šumpich, J. Liška), many others were observed. The voucher specimens were obtained by sweeping dry grass in sandy habitats. The species occurs in all parts of Europe (RAZOWSKI 2003, AARVIK in KARSHOLT \& NIEUKERKEN 2004) but is very local and it is unknown in many areas. For example, it is not known from the Moravian-Silesian region of the neighbouring Czech Republic (LAŠTƯVKA \& LIŠKA 2005). Although its very abundant occurrence was documented in Augustów Forest, it is generally a rare species in Poland. Recent records are only from the province of Warmia-Mazury (BUSZKO \& NOWACKI 2000).

\section{Epermenia falciformis (HAWORTH, 1828)}

(Epermeniidae)

\section{Material examined}

Białowieża env., Podolany village, 3 males (Fig. 18), 21 VI 2008 (leg., coll. J. Šumpich, J. Liška). This species was overlooked in the past, and it was not distinguished from Epermenia illigerella (HÜBNER, 1813) until recently (SCHOLZ 1996). Mainly on this account, its occurrence is still known from only a small number of countries, mostly in central and northern Europe (SCHOLZ 1996, GAEDIKE in KARSHOLT \& NIEUKERKEN 2004). Present-day records in neighbouring countries are from most German Lands (GAEDIKE \& HEINICKE 1999), Bohemia (NOVÁK et al. 1997), Moravia (ك̌UMPICH et al. 2006) and Slovakia (TOKÁR et al. 1999). To date, no evidence of its occurrence in Poland has been forthcoming. It is thus a new species for Poland.

\section{CONCLUSION}

In total nearly 700 moth species were found during three excursions to north-eastern Poland between 2000 and 2008. Among them were a number of faunistically or zoogeographically rare findings of interest beyond that of Polish entomofaunistics. Although we can regard the entire area of north-eastern Poland as having rather extraordinary natural conditions, here, too, we can observe the trend of decreasing insect populations that is characteristic of the whole of Europe. If we compare our impressions from our excursions in Poland with the findings obtained during our studies of the lepidopteran fauna of the Czech lands, however, we must state that the situation in Poland is still much more favourable than that in the Czech Republic (i.e. species abundances were conspicuously higher in comparable biotope types). We should note that the general decrease of moths in Europe, where a number of species are in jeopardy and in extreme cases even dying out, relates not only to large and attention-grabbing species but also to some very small species (the "Microlepidoptera" group) that are today very easily overlooked. 

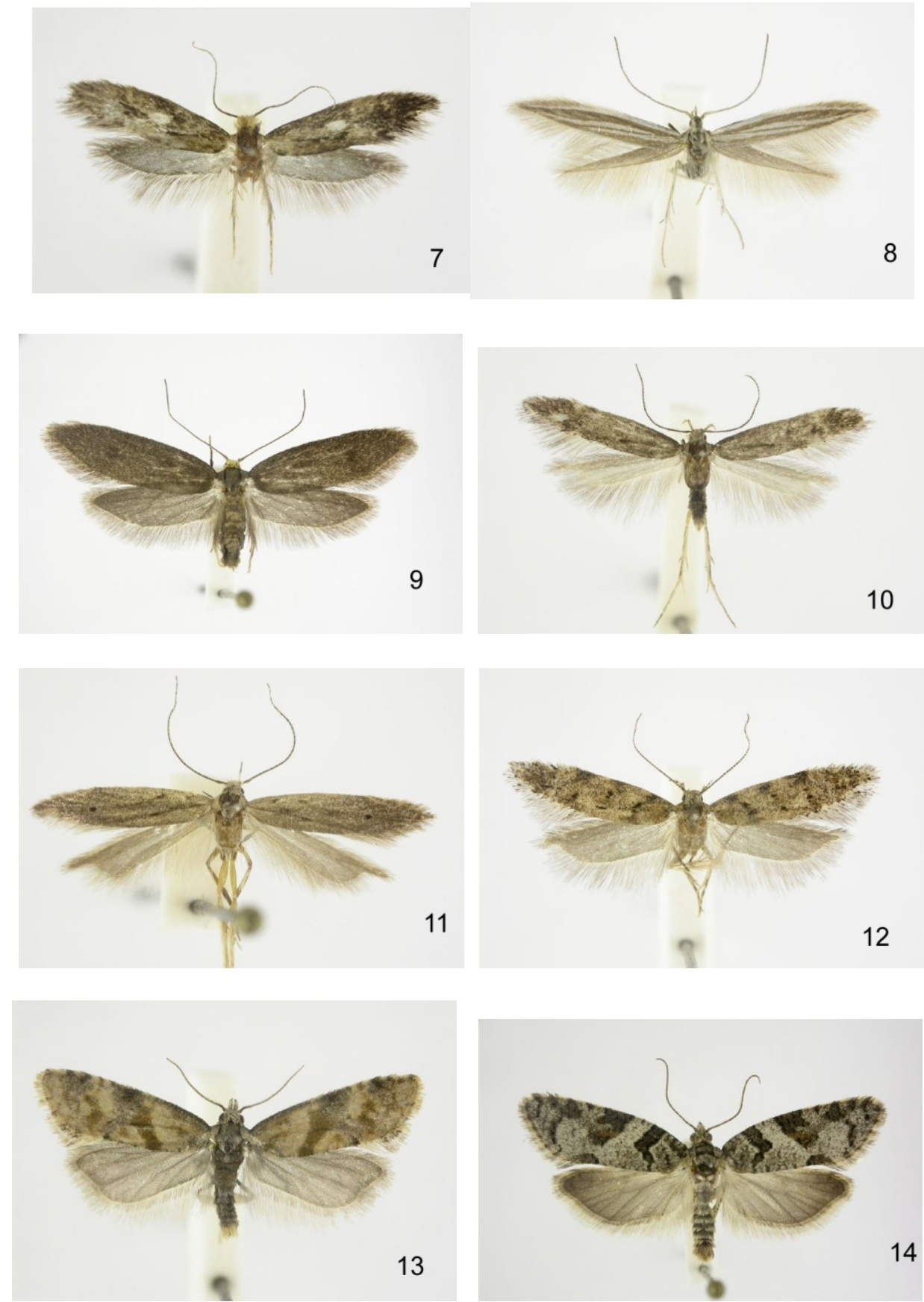

Figs. 7-14. Voucher specimens of some significant records: 7. Monopis fenestratella; 8. Coleophora ptarmicia; 9. Pseudatemelia elsae; 10. Monochroa rumicetella; 11. Monochroa niphognatha; 12. Carpatolechia aenigma; 13. Cochylidia moguntiana; 14. Eana derivana. 

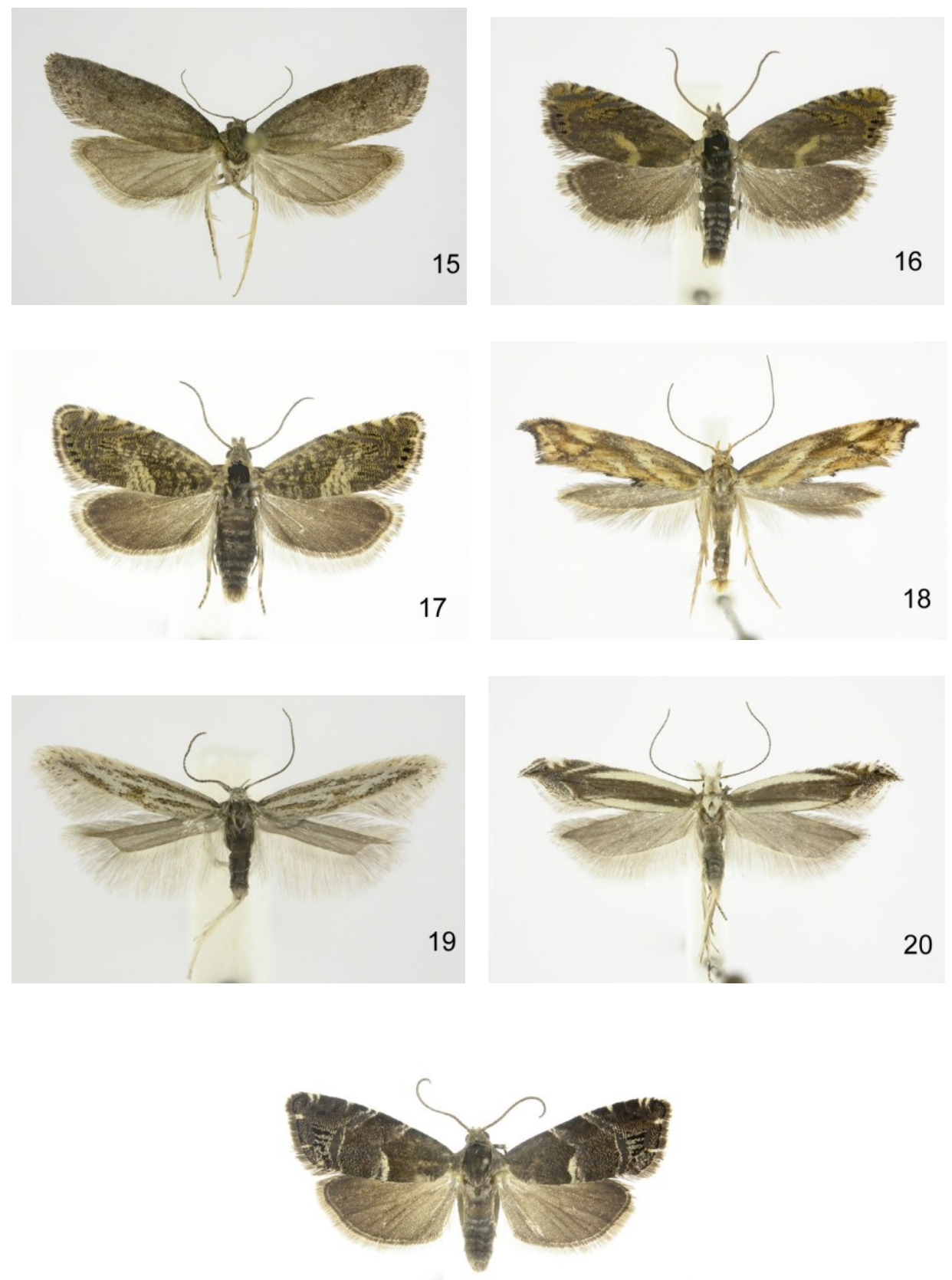

21

Figs 15-21. Voucher specimens of some significant records: 15. Cnephasia pasiuana; 16. Dichrorampha incognitana; 17. Dichrorampha agilana; 18. Epermenia falciformis; 19. Ptocheuusa inopella; 20. Sophronia chilonella; 21. Cydia coniferana. 


\section{REFERENCES}

Bengtsson B.Á. 1997. Scythrididae. [In:] Huemer P., Karsholt O. \& Lyneborg L. (eds). Microlepidoptera of Europe 2. Apollo Books, Stenstrup, $301 \mathrm{pp}$.

BidZILYA O.V., BudASHKIN YU.I. 2009. New records of Lepidoptera from Ukraine. Proceedings of the Zoological Museum 5: 14-28. [In Ukrainian].

Buszko J., NowAcki J. (eds). 2000. The Lepidoptera of Poland. A distributional Checklist. Polish Entomological Monographs 1, $178 \mathrm{pp}$.

Elsner G., Huemer P., TOKÁr Z. 1999. Die Palpenmotten (Lepidoptera, Gelechiidae) Mitteleuropas. Bestimmung, Verbreitung, Flugstandort, Lebensweise der Raupen. F. Slamka, Bratislava, 208 pp.

GaEdike R., HeINICKE W. (Hrsg.) 1999. Verzeichnis der Schmetterlinge Deutschlands (Entomofauna Germanica 3). Entomologische Nachrichten und Berichte (Dresden) Beiheft 5: 1-216.

GAEDIKE R., KARShOlt O. 2001. Contribution to the Lepidoptera fauna of the Madeira Islands. Part 2. Tineidae, Acrolepiidae, Epermeniidae. Beiträge zur Entomologie 51: 161-213.

GREgOR F., LAŠTU゚VKA A., LAŠTU゚VKA Z., MAREK J. 1984. Zur Verbreitung der Coleophora-Arten in der Tschechoslowakei (Lepidoptera, Coleophoridae). Biologia (Bratislava) 39: 1023-1032.

Karsholt O., Nieukerken E.J. van (eds). 2004. Fauna Europaea: Lepidoptera, Moths. Fauna Europaea, Version 2.1, http://www.faunaeur.org (visited on 15 March 2010).

KuZNETZOV V.I., BARYSCHNIKOVA S.V. 2003. A brief review of gracillariid moths of the genus Parornix Spuler, 1910 (Lepidoptera, Gracillariidae) of the Palearctic fauna. Entomologiceskoe Obozrenie 82: 116-137 [In Russian].

LAŠTU゚VKA Z., LIŠKA J. 2005. Checklist of Lepidoptera of the Czech Republic (Insecta: Lepidoptera). http://www.mendelu.cz/user/zooapi/lepidoptera.htm (visited on 15 March 2010).

LišKa J., LaštůVka Z., Jaroš J., Marek J., NĚMÝ J., Petrů M., Elsner G., Skyva J., FranZ J. 2001. Faunistic records from the Czech Republic - 142. Klapalekiana 37: 275-278.

Novák I., LišKa J., Elsner G., Jaroš J., Petrů M., Skyva J., Spitzer K., Špatenka K., VÁvra J., WEIDENHOFFER Z. 1997. Katalog motýlů (Lepidoptera) Čech. Klapalekiana 33 (Suppl.): 1-159.

Petersen G. 1957. Die Genitalien der paläarktischen Tineiden (Lepidoptera: Tineidae). Beiträge zur Entomologie 7: 55-176.

PETERSEN G. 1969. Beiträge zur Insekten-Fauna der DDR: Lepidoptera - Tineidae. Beiträge zur Entomologie 19: 311-388.

Petersen G., Gaedike R. 1987. Beiträge zur Insektenfauna der DDR: Lepidoptera. Nachträge IV (Tineidae, Acrolepiidae, Crambidae, Galleriidae, Pyralidae s. str., Douglasiidae, Pyraustinae, Scopariinae, Phycitinae). Entomologische Nachrichten und Berichte 31: 29-36.

De PRINS W., De Prins J. 2005. Gracillariidae (Lepidoptera). World Catalogue of Insects 6: 1-502.

RAzowsKi J. 2002. Tortricidae of Europe. Volume 1. Tortricinae and Chlidanotinae. František Slamka, Bratislava, $247 \mathrm{pp}$.

RAzOWSKi J. 2003. Tortricidae of Europe. Volume 2. Olethreutinae. František Slamka, Bratislava, $301 \mathrm{pp}$.

REIPRICH A., OKÁLI I. 1989. Dodatky k Prodromu Lepidopter Slovenska. 2. zväzok (Ergänzungen zu Prodromus Lepidopterum Slovakiae). 2. Teil. Veda, vydavatel'stvo Slovenskej akadémie vied, Bratislava, 107 pp [in Slovak, German abstr.].

Robinson G.S. 2002. Global Taxonomic Database of Tineidae (Lepidoptera). http://www.nhm.ac.uk/research-curation/research/projects/tineidae (visited 15 March 2010).

Scholz A. 1996. Zur Identität von Epermenia falciformis (Haworth, 1828) (Lepidoptera: Epermeniidae). Nota Lepidopterologica 18: 289-296.

SiteK J. 2008. Faunistic records from the Czech Republic - 251. Klapalekiana 44: 75-76.

SteRneck J., ZimmermanN F. 1933: Prodromus der Schmetterlingsfauna Böhmens II. (Microlepidoptera). Jacob Sterneck, Karlsbad, 168 pp. 
ŠUMPICH J., LišKA J., Elsner G., ŽEMLIČKA M., MAREK J., DvoŘÁK I., DvořÁK M., DOBROvskÝ T., SKYVA J. 2006. Faunistic records from the Czech Republic - 202. Klapalekiana 42: 181-187.

Šumpich J., ŽEmličKa M., Č́́la P., Heřman P., LišKa J., Elsner G., MAReK J., LAŠTŮVKa A., SKYVA J., MikÁt M., Rotter M. 2007. Faunistic records from the Czech Republic - 225. Klapalekiana 43: 79-84.

TokÁr Z., Lvovsky A., Huemer, P. 2005. Die Oecophoridae s. 1. Mitteleuropas (Lepidoptera) Bestimmung, Verbreitung, Habitat, Bionomie. František Slamka, Bratislava, 120 pp.

TokÁr Z., Richter I., PAstorális G., Slamka F. 2002. New and interesting records of Lepidoptera of Slovakia from the years 1998-2001. Entomofauna Carphatica 14: 1-11.

TokÁr Z., Slamka F., Pastorális G. 1999. New and interesting records of Lepidoptera from Slovakia in 1995-1997. Entomofauna Carpathica 11: 43-57.

VlaCH V. 1936. Microlepidoptera pro Čechy nová nebo pochybná. Čas. Česke Společnosti Entomologičke 33: 18-19 [In Czech].

Xiao Y., Li H. 2006. A review of the genus Monopis Hübner from China (Lepidoptera, Tineidae, Tineinae). Mitteilungen aus dem Museum für Naturkunde in Berlin; Deutsche. Entomologische Zeitschrift 53: 193-212.

ZagulJaEv A.K. 1960. Tineidae; part 3, subfamily Tineinae. Fauna SSSR 78: 1-267 (in Russian).

ZimMERMANN F., SkALA H. 1947. Kleinfalter aus Mähren-Schlesien. Zeitschrift der Wiener Entomologischen Gesellschaft 31: 121-123.

\section{APPENDIX}

Overview of species of butterflies and moths found in Podlasie in 2000-2008.

(Explanations: numerals in columns 2-6 represent the numbers of specimens, species in column 7 (other) were observed to the amount of 10 specimens; the "other" column - 1) Strękowizna 15 VI 2008, 2) Sucha Rzeczka 18 VI 2008, 3) Strzelcowizna 18 VI 2008; "Notes" column: PL - new species for Poland, Pd - new species for Podlasie, (!) confirmation of occurrence after a long time).

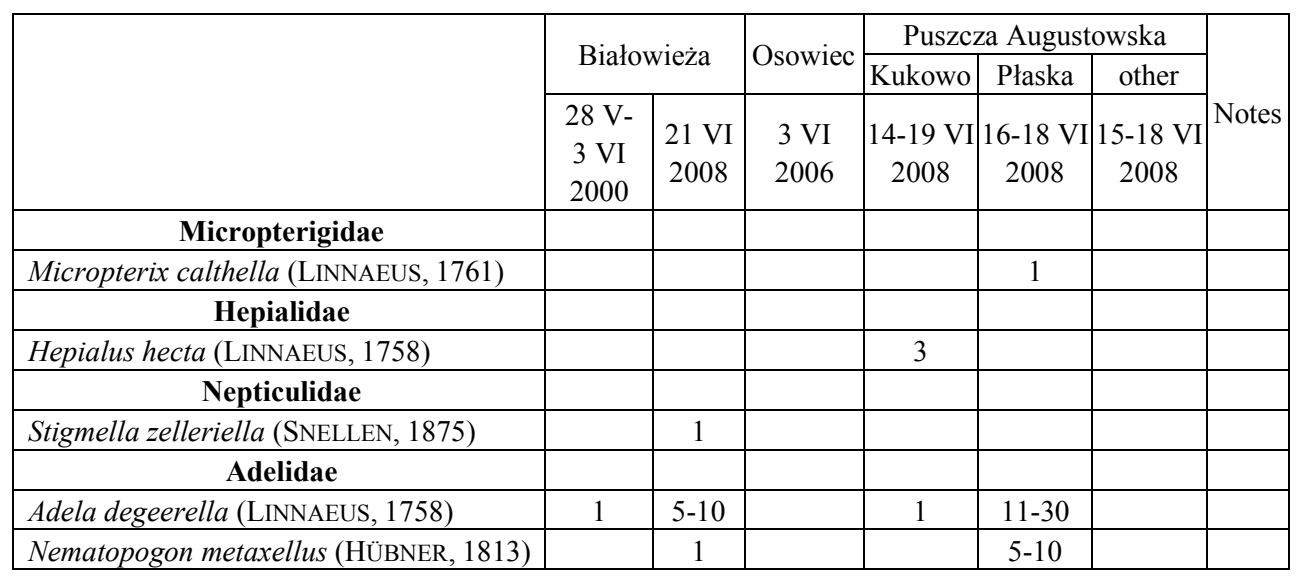




\begin{tabular}{|c|c|c|c|c|c|c|}
\hline Incurvariidae & & & & & & \\
\hline Incurvaria oehlmanniella (HÜBNER, 1796) & 1 & & & & & \\
\hline $\begin{array}{l}\text { Incurvaria praelatella (DEN. \& SCHIFF., } \\
1775 \text { ) }\end{array}$ & & 1 & & $5-10$ & 1 & \\
\hline Lampronia corticella (LINNAEUS, 1758) & 1 & & & & & \\
\hline \multicolumn{7}{|l|}{ Tischeriidae } \\
\hline Emmetia marginea (HAWORTH, 1828) & & & & & 1 & $\mathrm{Pd}$ \\
\hline \multicolumn{7}{|l|}{ Tineidae } \\
\hline Haplotinea insectella (FABRICIUS, 1794) & 1 & & & & & \\
\hline Scardia boletella (FABRICIUS, 1794) & & & 1 & & & \\
\hline $\begin{array}{l}\text { Triaxomera fulvimitrella (SODOFFSKY, } \\
1830 \text { ) }\end{array}$ & 1 & & & & & $\mathrm{Pd}$ \\
\hline Triaxomera parasitella (HÜBNER, 1796) & 1 & & 1 & & & \\
\hline Archinemapogon yildizae КОСАК, 1981 & & & 1 & & & \\
\hline Nemaxera betulinella (PAYKULL, 1785) & & & & & 1 & $\mathrm{Pd}$ \\
\hline Nemapogon cloacellus (HAWORTH, 1828) & 2 & 1 & 1 & & 1 & \\
\hline $\begin{array}{l}\text { Nemapogon wolffiellus KARS.\& NIELSEN, } \\
1976\end{array}$ & & 1 & 1 & & & $\mathrm{Pd}$ \\
\hline Tinea trinotella THUNBERG, 1794 & & & & & 1 & \\
\hline Monopis obviella (DEN. \& SCHIFF., 1775) & & & & & 1 & \\
\hline Monopis monachella (HÜBNER, 1796) & 1 & & & & 1 & \\
\hline Monopis fenestratella (HEYDEN, 1863) & & & & & 1 & PL \\
\hline Monopis bisonella sp. $\mathrm{n}$. & 1 & & & & & sp. n. \\
\hline \multicolumn{7}{|l|}{ Psychidae } \\
\hline Taleporia tubulosa (RETZIUS, 1783) & 1 & & 1 & & & \\
\hline Proutia betulina (ZELLER, 1839) & & 1 & & & 1 & \\
\hline Psyche casta (PALLAS, 1767) & & 2 & & & & \\
\hline $\begin{array}{l}\text { Bijugis bombycella (DEN. \& SCHIFF., } \\
\text { 1775) }\end{array}$ & 3 & & & & & \\
\hline Sterrhopterix fusca (HAWORTH, 1809) & $5-10$ & & & 1 & $11-30$ & \\
\hline \multicolumn{7}{|l|}{ Bucculatricidae } \\
\hline $\begin{array}{l}\text { Bucculatrix bechsteinella (BCH.\& } \\
\text { SCHARF., 1805) }\end{array}$ & & & & & 1 & \\
\hline Bucculatrix frangutella (GOEZE, 1783) & & & & & 1 & \\
\hline \multicolumn{7}{|l|}{ Gracillariidae } \\
\hline Caloptilia populetorum (ZELLER, 1839) & 3 & & & & 1 & \\
\hline Caloptilia alchimiella $($ SCOPOLI, 1763) & & & & 1 & 2 & \\
\hline Caloptilia stigmatella (FABRICIUS, 1781) & & & & & 1 & \\
\hline $\begin{array}{l}\text { Aspilapteryx tringipennella (ZELLER, } \\
\text { 1839) }\end{array}$ & & & & & 1 & $\mathrm{Pd}$ \\
\hline $\begin{array}{l}\text { Phyllonorycter kleemannellus } \\
\text { (FABRICIUS, 1781) }\end{array}$ & & 1 & & & 1 & $\mathrm{Pd}$ \\
\hline Parornix avellanella (STAINTON, 1854) & & & & 2 & & $\mathrm{Pd}$ \\
\hline Phyllocnistis saligna (ZELLER, 1839) & & & & & 1 & $\mathrm{Pd}$ \\
\hline \multicolumn{7}{|l|}{ Yponomeutidae } \\
\hline $\begin{array}{l}\text { Swammerdamia compunctella (HER.- } \\
\text { SCH., 1855) }\end{array}$ & & & & 1 & & \\
\hline
\end{tabular}




\begin{tabular}{|c|c|c|c|c|c|c|c|}
\hline Cedestis gysseleniella ZELLER, 1839 & & & & & 1 & & \\
\hline Cedestis subfasciella (STEPHENS, 1834) & & & & & 1 & & $\mathrm{Pd}$ \\
\hline Ocnerostoma piniariellum ZELLER, 1847 & & & & & 1 & 1) & \\
\hline Prays fraxinellus (BJERKANDER, 1784) & 1 & & & & & & \\
\hline Argyresthia glabratella (ZELLER, 1847) & & & & & 1 & & \\
\hline Argyresthia bergiella (RATZEBURG, 1840 ) & & & & 1 & & & $\mathrm{Pd}$ \\
\hline Argyresthia abdominalis ZELLER, 1839 & & & & & $5-10$ & & $\mathrm{Pd}$ \\
\hline Argyresthia brockeella (HÜBNER, 1813) & & & & & 2 & & \\
\hline Argyresthia curvella (LINNAEUS, 1761) & & & & & 1 & & $\mathrm{Pd}$ \\
\hline Argyresthia retinella ZELLER, 1839 & & & & & $11-30$ & & \\
\hline Argyresthia conjugella ZELLER, 1839 & & & & & 1 & & \\
\hline \multicolumn{8}{|l|}{ Plutellidae } \\
\hline Plutella xylostella (LINNAEUS, 1758) & 1 & & & & 1 & & \\
\hline \multicolumn{8}{|l|}{$\begin{array}{c}\text { Glyphipterigidae } \\
\end{array}$} \\
\hline Glyphipterix thrasonella (SCOPOLI, 1763) & 1 & & & & & & \\
\hline Glyphipterix equitella (SCOPOLI, 1763) & & & & 1 & & & \\
\hline $\begin{array}{l}\text { Glyphipterix forsterella (FABRICIUS, } \\
\text { 1781) }\end{array}$ & & & & 1 & & & \\
\hline \multicolumn{8}{|l|}{$\begin{array}{c}\text { Ethmiidae } \\
\end{array}$} \\
\hline Ethmia quadrillella (GOEZE, 1783) & $5-10$ & & & & & & \\
\hline \multicolumn{8}{|l|}{ Depressariidae } \\
\hline Luquetia lobella (DEN. \& SCHIFF., 1775) & $5-10$ & & & 2 & 1 & & $\mathrm{Pd}$ \\
\hline Agonopterix ocellana (FABRICIUS, 1775) & & & & & 1 & & \\
\hline $\begin{array}{l}\text { Agonopterix arenella (DEN. \& SCHIFF., } \\
\text { 1775) }\end{array}$ & & & & & 1 & & \\
\hline \multicolumn{8}{|l|}{ Oecophoridae } \\
\hline Denisia similella (HÜBNER, 1796) & 1 & & & & $11-30$ & & \\
\hline Denisia stipella (LINNAEUS, 1758) & & & & & 1 & & \\
\hline Metalampra cinnamomea (ZELLER, 1839) & & & & & 1 & & \\
\hline Borkhausenia minutella (LINNAEUS, 1758) & & & 1 & & & & \\
\hline Crassa tinctella (HÜBNER, 1796) & & & & & 1 & & \\
\hline Pleurota bicostella (CLERCK, 1759) & & & 1 & & 1 & & \\
\hline \multicolumn{8}{|l|}{ Elachistidae } \\
\hline Mendesia farinella (THUNBERG, 1794) & & & $5-10$ & & $11-30$ & & \\
\hline Elachista utonella FREY, 1856 & & & & 3 & & & $\mathrm{Pd}$ \\
\hline Elachista albidella NYLANDER, 1848 & & & & & 2 & & \\
\hline Elachista albifrontella (HÜBNER, 1817) & & & & $11-30$ & $5-10$ & & \\
\hline Elachista humilis ZELLER, 1850 & & & & 3 & & & $\mathrm{Pd}$ \\
\hline Elachista maculicerusella BRUAND, 1859 & & 1 & & $11-30$ & & 1) & \\
\hline Elachista argentella $($ CLERCK, 1759) & & & & & 2 & & \\
\hline Elachista pollinariella ZELLER, 1839 & & & & $11-30$ & $11-30$ & 1) & $\mathrm{Pd}$ \\
\hline Elachista pullicomella ZELLER, 1839 & & & 2 & & & & $\mathrm{Pd}$ \\
\hline \multicolumn{8}{|l|}{$\begin{array}{c}\text { Agonoxenidae } \\
\end{array}$} \\
\hline Blastodacna atra (HAWORTH, 1828) & & & & & 1 & & $\mathrm{Pd}$ \\
\hline \multicolumn{8}{|l|}{ Scythrididae } \\
\hline Scythris palustris (ZELLER, 1855) & 1 & & & & & & $\mathrm{Pd}$ \\
\hline
\end{tabular}




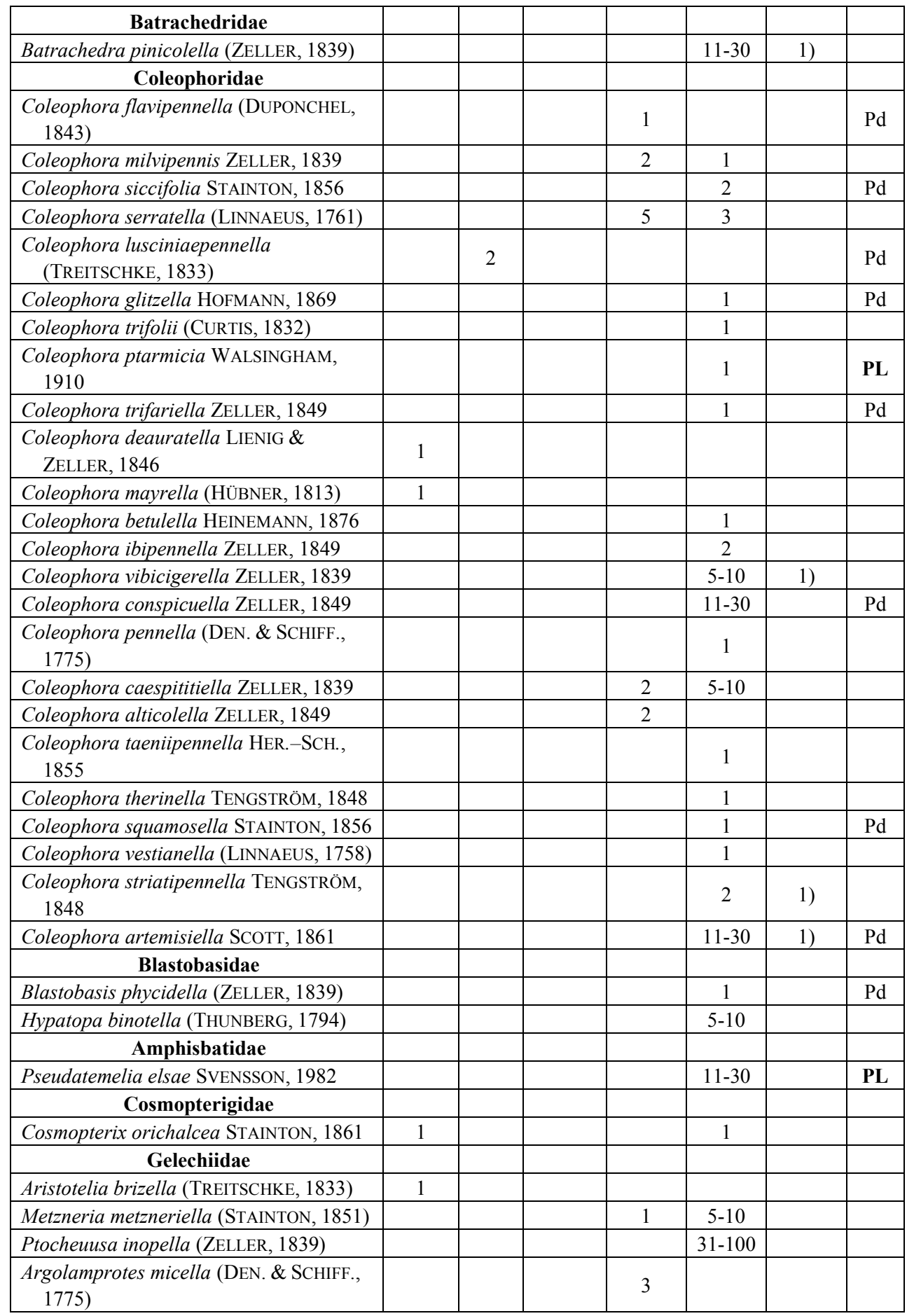




\begin{tabular}{|c|c|c|c|c|c|c|c|}
\hline Monochroa rumicetella (HOFMANN, 1868) & & & 2 & & & & $\mathrm{Pd}$ \\
\hline Monochroa servella (ZELLER, 1839) & & & & & 1 & & PL (!) \\
\hline $\begin{array}{l}\text { Monochroa niphognatha (GOZMÁNY, } \\
\text { 1953) }\end{array}$ & 1 & & & & & & \\
\hline Eulamprotes wilkella (LINNAEUS, 1758) & & & & & & 1) & \\
\hline Eulamprotes superbella (ZELLER, 1839) & & & 4 & & & & $\mathrm{Pd}$ \\
\hline $\begin{array}{l}\text { Eulamprotes unicolorella (DUPONCHEL, } \\
\text { 1843) }\end{array}$ & & & & & $5-10$ & & $\mathrm{Pd}$ \\
\hline $\begin{array}{l}\text { Bryotropha terrella (DEN. \& SCHIFF., } \\
\text { 1775) }\end{array}$ & & & & & $5-10$ & & \\
\hline Bryotropha desertella (DOUGLAS, 1850) & & & & & 2 & & \\
\hline Bryotropha similis (STAINTON, 1854) & & & & & $11-30$ & 1) & \\
\hline Exoteleia dodecella (LINNAEUS, 1758) & & & & 1 & $>100$ & & \\
\hline Parachronistis albiceps (ZELLER, 1839) & & & & & 1 & & \\
\hline Teleiodes luculellus (HÜBNER, 1813) & 1 & & & & 1 & & \\
\hline Teleiodes wagae (NowICKI, 1860) & 3 & & 1 & & & & \\
\hline Carpatolechia alburnellus (ZELLER, 1839) & & & & & 1 & & \\
\hline $\begin{array}{l}\text { Pseudotelphusa paripunctella } \\
\text { (THUNBERG, 1794) }\end{array}$ & & & $11-30$ & & & & \\
\hline Carpatolechia proximella (HÜBNER, 1796) & & 1 & 3 & 1 & & & \\
\hline Carpatolechia aenigma SATTLER, 1983 & & & & & 1 & & PL (!) \\
\hline Teleiopsis diffinis (HAWORTH, 1828) & & & 2 & & 1 & & $\operatorname{Pd}(!)$ \\
\hline Pseudotelphusa scalella (SCOPOLI, 1763) & & & & & $5-10$ & & \\
\hline Altenia scriptella (HÜBNER, 1796) & & & & 1 & & & \\
\hline Gelechia sabinella (ZELLER, 1839) & & & & & 1 & & $\mathrm{Pd}$ \\
\hline Chionodes luctuellus (HÜBNER, 1793) & & & & $5-10$ & & & \\
\hline Neofriseria peliella (TREITSCHKE, 1835) & & & & & $5-10$ & & \\
\hline $\begin{array}{l}\text { Euscrobipalpa acuminatella (SIRCOM, } \\
\text { 1850) }\end{array}$ & & & 1 & & & & \\
\hline Sophronia semicostella $($ HÜBNER, 1813) & & & & & $31-100$ & 1) & \\
\hline Sophronia chilonella (TREITSCHKE, 1833) & & & & & $31-100$ & 1) & $\mathrm{Pd}$ \\
\hline $\begin{array}{l}\text { Sophronia humerella (DEN. \& SCHIFF., } \\
\text { 1775) }\end{array}$ & & & & & 1 & & \\
\hline Syncopacma sangiella (STAINTON, 1863) & 4 & & & & & & $\mathrm{Pd}$ \\
\hline Neofaculta infernella (HER.-SCH., 1854) & 1 & & $31-100$ & & & & \\
\hline Neofaculta ericetella (GEYER, 1832) & & & $11-30$ & & $5-10$ & & \\
\hline Dichomeris marginella (FABRICIUS, 1781) & & & & 1 & & & $\mathrm{Pd}$ \\
\hline Dichomeris ustalella (FABRICIUS, 1794) & 1 & & & & & & \\
\hline Dichomeris limosella (SCHLÄGER, 1849) & & 1 & & & & & PL (!) \\
\hline $\begin{array}{l}\text { Brachmia dimidiella (DEN. \& SCHIFF., } \\
\text { 1775) }\end{array}$ & & & & & & 1) & \\
\hline Brachmia blandella (FABRICIUS, 1798) & & & & & 1 & & \\
\hline Brachmia inornatella (DOUGLAS, 1850) & & & & & 1 & & \\
\hline $\begin{array}{l}\text { Helcystogramma lutatellum (HER.-SCH., } \\
\text { 1854) }\end{array}$ & & & & 1 & & 1) & \\
\hline $\begin{array}{l}\text { Helcystogramma rufescens (HAWORTH, } \\
\text { 1828) }\end{array}$ & 1 & & & 1 & 1 & & \\
\hline
\end{tabular}




\begin{tabular}{|c|c|c|c|c|c|c|}
\hline $\begin{array}{l}\text { Helcystogramma albinerve (GERASIMOV, } \\
\text { 1929) }\end{array}$ & 1 & & & & 3 & \\
\hline Acompsia cinerella (CLERCK, 1759) & & & & 1 & $5-10$ & \\
\hline \multicolumn{7}{|l|}{$\begin{array}{c}\text { Limacodidae } \\
\end{array}$} \\
\hline Apoda limacodes (HUFNAGEL, 1766) & & & & & $5-10$ & \\
\hline \multicolumn{7}{|l|}{$\begin{array}{c}\text { Zygaenidae } \\
\end{array}$} \\
\hline Adscita statices (LINNAEUS, 1758) & $31-100$ & & & & & \\
\hline \multicolumn{7}{|l|}{ Cossidae } \\
\hline $\begin{array}{l}\text { Phragmataecia castaneae (HÜBNER, } \\
1790)\end{array}$ & & & & & 1 & \\
\hline Cossus cossus (LINNAEUS, 1758) & $5-10$ & & & & 1 & \\
\hline \multicolumn{7}{|l|}{$\begin{array}{c}\text { Tortricidae } \\
\end{array}$} \\
\hline Phtheochroa inopiana (HAWORTH, 1811) & 1 & & & & & \\
\hline Phtheochroa sodaliana (HAWORTH, 1811) & & & & & 2 & \\
\hline $\begin{array}{l}\text { Phalonidia manniana (FISCHER V.R., } \\
\text { 1839) }\end{array}$ & $11-30$ & & & & 1 & \\
\hline $\begin{array}{l}\text { Gynnidomorpha permixtana (DEN. \& } \\
\text { SCHIFF., 1775) }\end{array}$ & 2 & & & & 1 & \\
\hline Agapeta hamana (LINNAEUS, 1758) & & & & 1 & & \\
\hline Aethes hartmanniana (CLERCK, 1759) & & & & & 2 & \\
\hline Aethes margaritana (HAWORTH, 1811) & & & & & 2 & \\
\hline Aethes triangulana (TREITSCHKE, 1835) & & 2 & & & & \\
\hline Aethes rutilana (HÜBNER, 1817) & & & & & 2 & PL $(!)$ \\
\hline $\begin{array}{l}\text { Aethes smeathmanniana (FABRICIUS, } \\
\text { 1781) }\end{array}$ & & & 1 & 1 & & \\
\hline Aethes cnicana (WESTWOOD, 1854) & & & & 1 & & $\mathrm{Pd}$ \\
\hline Aethes rubigana (TREITSCHKE, 1830) & $11-30$ & & & & & \\
\hline Cochylidia heydeniana (HER.-SCH., 1851) & & & & & 1 & $\mathrm{Pd}$ \\
\hline Cochylidia moguntiana (RÖSSLER, 1864) & & & & & $11-30$ & $\mathrm{Pd}$ \\
\hline Cochylidia implicitana (WOCKE, 1856) & & & & & $5-10$ & \\
\hline Cochylis flaviciliana (WESTWOOD, 1854) & & & & & 1 & \\
\hline Cochylis dubitana (HÜBNER, 1799) & 2 & & & & & \\
\hline Tortrix viridana (LINNAEUS, 1758) & $5-10$ & & & & & \\
\hline Aleimma loeflingianum (LINNAEUS, 1758) & 1 & & & & 1 & \\
\hline $\begin{array}{l}\text { Acleris comariana (LIENIG \& ZELLER, } \\
\text { 1846) }\end{array}$ & & & & & 1 & \\
\hline Acleris laterana (FABRICIUS, 1794) & & & & & 1 & \\
\hline Eana derivana (LA HARPE, 1858) & & & & & $11-30$ & PL $(!)$ \\
\hline Cnephasia communana (HER.-SCH., 1851) & & & & 1 & & \\
\hline $\begin{array}{l}\text { Cnephasia asseclana (DEN. \& SCHIFF., } \\
\text { 1775) }\end{array}$ & 1 & 2 & & 2 & & \\
\hline Cnephasia incertana (TREITSCHKE, 1835) & 1 & & & $11-30$ & & $\mathrm{Pd}$ \\
\hline Cnephasia pasiuana (HÜBNER, 1799) & & & & & 2 & $\mathrm{Pd}$ \\
\hline Eulia ministrana (LINNAEUS, 1758) & $5-10$ & & 1 & 1 & 1 & \\
\hline Epagoge grotiana (FABRICIUS, 1781) & & 2 & & & & \\
\hline Paramesia gnomana (CLERCK, 1759) & & 1 & & & & \\
\hline Capua vulgana (FRÖLICH, 1828) & 1 & & & & 1 & \\
\hline
\end{tabular}




\begin{tabular}{|c|c|c|c|c|c|c|c|}
\hline Archips podanus (SCOPOLI, 1763) & $11-30$ & & & & 1 & & \\
\hline Archips xylosteanus (LINNAEUS, 1758) & & & & & 2 & & \\
\hline $\begin{array}{l}\text { Choristoneura hebenstreitella (MÜLLER, } \\
\text { 1764) }\end{array}$ & 1 & & & & & & \\
\hline Ptycholoma lecheanum (LINNAEUS, 1758) & 1 & & & & & & \\
\hline Pandemis corylana (FABRICIUS, 1794) & 1 & & & & 1 & & \\
\hline Pandemis cerasana (HÜBNER, 1786) & 1 & & & 1 & $5-10$ & & $\mathrm{Pd}$ \\
\hline $\begin{array}{l}\text { Pandemis heparana (DEN. \& SCHIFF., } \\
\text { 1775) }\end{array}$ & 1 & & & & & & \\
\hline Lozotaenia forsterana (FABRICIUS, 1781) & & & & 1 & 3 & & \\
\hline Aphelia unitana (HÜBNER,1799) & $5-10$ & $5-10$ & & & & & $\mathrm{Pd}$ \\
\hline Dichelia histrionana (FRÖLICH, 1828) & & & & 4 & 1 & & $\mathrm{Pd}$ \\
\hline Clepsis spectrana (TREITSCHKE, 1830) & 1 & & & $11-30$ & & & \\
\hline Bactra lancealana (HÜBNER, 1799) & & $5-10$ & & $11-30$ & $5-10$ & & \\
\hline Bactra furfurana (HAWORTH, 1811) & 1 & & & & & & \\
\hline $\begin{array}{l}\text { Endothenia nigricostana (HAWORTH, } \\
\text { 1811) }\end{array}$ & 1 & & & & & & $\mathrm{Pd}$ \\
\hline $\begin{array}{l}\text { Endothenia quadrimaculana (HAWORTH, } \\
\text { 1811) }\end{array}$ & 1 & & & & & & \\
\hline $\begin{array}{l}\text { Pseudosciaphila branderiana (LINNAEUS, } \\
1758 \text { ) }\end{array}$ & & & & 1 & & & \\
\hline Apotomis semifasciana (HAWORTH, 1811) & 2 & & & 2 & 1 & & $\mathrm{Pd}$ \\
\hline Apotomis turbidana HÜBNER, 1825 & $5-10$ & & & & $5-10$ & & \\
\hline Apotomis betuletana (HAWORTH, 1811) & & & & 1 & $5-10$ & & \\
\hline Apotomis capreana $($ HÜBNER, 1817) & & 1 & & 1 & 1 & & \\
\hline Apotomis sauciana (FRÖLICH, 1828) & & & & & 1 & & $\mathrm{Pd}$ \\
\hline $\begin{array}{l}\text { Orthotaenia undulana (DEN. \& SCHIFF., } \\
\text { 1775) }\end{array}$ & & $5-10$ & & 2 & & & \\
\hline Hedya salicella (LINNAEUS, 1758) & $5-10$ & & & & & & \\
\hline Hedya nubiferana (HAWORTH, 1811) & 1 & & & $11-30$ & $5-10$ & & \\
\hline Hedya pruniana (HÜBNER, 1799) & & & 1 & & & & \\
\hline Hedya dimidiana (CLERCK, 1759) & 2 & & & & & & $\mathrm{Pd}$ \\
\hline $\begin{array}{l}\text { Metendothenia atropunctana } \\
\text { (ZETTERSTEDT, 1839) }\end{array}$ & 1 & & 1 & & $5-10$ & & \\
\hline Celypha rufana (SCOPOLI, 1763) & & & & & $5-10$ & 1) & \\
\hline Celypha striana (DEN. \& SCHIFF., 1775) & & & & 1 & 2 & & \\
\hline Celypha cespitana (HÜBNER, 1817) & & & & & 1 & & \\
\hline Celypha lacunana (DEN. \& SCHIFF., 1775) & 1 & $11-30$ & $5-10$ & $5-10$ & & & \\
\hline Celypha siderana (TREITSCHKE, 1835) & & & & & 1 & & $\operatorname{Pd}(!)$ \\
\hline Celypha rivulana (SCOPOLI, 1763) & $5-10$ & & & & & & \\
\hline Olethreutes arcuellus (CLERCK, 1759) & 2 & & & & 1 & & \\
\hline Olethreutes umbrosanus (FREYER, 1842) & 1 & & 1 & 1 & & & \\
\hline $\begin{array}{l}\text { Olethreutes bipunctanus (FABRICIUS, } \\
\text { 1794) }\end{array}$ & & & $11-30$ & 3 & & & \\
\hline Piniphila bifasciana (HAWORTH, 1811) & & & & & $11-30$ & & \\
\hline $\begin{array}{l}\text { Pseudohermenias abietana (FABRICIUS, } \\
1787 \text { ) }\end{array}$ & & & & 1 & & & \\
\hline
\end{tabular}




\begin{tabular}{|c|c|c|c|c|c|c|c|}
\hline Thiodia citrana (HÜBNER, 1799) & & & & & 1 & & \\
\hline Spilonota laricana (HEINEMANN, 1863) & & & & & 1 & & \\
\hline Epinotia trigonella (LINNAEUS, 1758) & & & & & 1 & & \\
\hline Epinotia abbreviana (FABRICIUS, 1794) & & & & & 1 & & \\
\hline Epinotia bilunana (HAWORTH, 1811) & & & & & 1 & & \\
\hline $\begin{array}{l}\text { Epinotia demarniana (FISCHER V.R., } \\
1840 \text { ) }\end{array}$ & & & & & 1 & & \\
\hline $\begin{array}{l}\text { Epinotia immundana (FISCHER V.R., } \\
\text { 1839) }\end{array}$ & 1 & & & & & & \\
\hline Epinotia tetraquetrana $(\mathrm{HAWORTH}, 1811)$ & & & 1 & & & & \\
\hline Epinotia tenerana (DEN. \& SCHIFF., 1775) & & 1 & & & & & \\
\hline Epinotia tedella (CLERCK, 1759) & 1 & & 1 & 1 & $11-30$ & & \\
\hline Epinotia rubiginosana (HER.-SCH., 1851) & & & & & $5-10$ & & $\mathrm{Pd}$ \\
\hline Epinotia cruciana (LINNAEUS, 1761) & & 1 & & & & & \\
\hline Epinotia nanana (TREITSCHKE, 1835) & & & & & 1 & & \\
\hline Eucosma cana (HAWORTH, 1811) & $5-10$ & & & & & & \\
\hline $\begin{array}{l}\text { Eucosma metzneriana (TREITSCHKE, } \\
1830 \text { ) }\end{array}$ & $11-30$ & & & & $5-10$ & & \\
\hline Gypsonoma dealbana (FRÖLICH, 1828) & 1 & & & & 2 & & \\
\hline Epiblema foenellum (LINNAEUS, 1758) & 1 & & & & & & $\mathrm{Pd}$ \\
\hline Epiblema sticticanum (FABRICIUS, 1794) & 2 & & & & & & \\
\hline $\begin{array}{l}\text { Epiblema scutulanum (DEN. \& SCHIFF., } \\
1775 \text { ) }\end{array}$ & & & & 1 & & & $\mathrm{Pd}$ \\
\hline $\begin{array}{l}\text { Epiblema graphanum (TREITSCHKE, } \\
\text { 1835) }\end{array}$ & & & & & $5-10$ & 1) & \\
\hline Notocelia cynosbatella (LINNAEUS, 1758) & & & & 1 & & & \\
\hline $\begin{array}{l}\text { Notocelia uddmanniana (LINNAEUS, } \\
1758 \text { ) }\end{array}$ & 1 & 1 & & & 1 & & \\
\hline Notocelia trimaculana $($ HAWORTH, 1811$)$ & 1 & & & 1 & & & $\mathrm{Pd}$ \\
\hline Retinia resinella (LINNAEUS, 1758) & & & & & $5-10$ & & \\
\hline $\begin{array}{l}\text { Rhyacionia buoliana (DEN. \& SCHIFF., } \\
\text { 1775) }\end{array}$ & & & & & $11-30$ & 1) & \\
\hline $\begin{array}{l}\text { Rhyacionia pinicolana (DOUBLEDAY, } \\
\text { 1849) }\end{array}$ & & & & & $11-30$ & & \\
\hline $\begin{array}{l}\text { Rhyacionia pinivorana (LIENIG \& } \\
\text { ZELLER, 1846) }\end{array}$ & & & 1 & & $>100$ & & \\
\hline Ancylis laetana (FABRICIUS, 1775) & 1 & & & & 1 & & \\
\hline Ancylis unculana (HAWORTH, 1811) & $5-10$ & 1 & 1 & $5-10$ & 2 & & \\
\hline Ancylis uncella (DEN. \& SCHIFF., 1775) & & & $5-10$ & & 2 & & $\mathrm{Pd}$ \\
\hline $\begin{array}{l}\text { Ancylis mitterbacheriana (DEN.\& SCH., } \\
\text { 1775) }\end{array}$ & $5-10$ & & & & $31-100$ & & \\
\hline Ancylis upupana (TREITSCHKE, 1835) & 2 & & & & & & \\
\hline Ancylis geminana (DONOVAN, 1806) & 1 & & & 1 & 1 & & \\
\hline Ancylis diminutana (HAWORTH, 1811) & & & 1 & 1 & & & $\mathrm{Pd}$ \\
\hline Ancylis achatana (DEN. \& SCHIFF., 1775) & & & $5-10$ & 1 & 1 & & \\
\hline Ancylis badiana (DEN. \& SCHIFF., 1775) & & & 1 & & & & \\
\hline Ancylis paludana (BARRETT, 1871) & & & 1 & & & & \\
\hline
\end{tabular}




\begin{tabular}{|c|c|c|c|c|c|c|c|}
\hline Ancylis apicella (DEN. \& SCHIFF., 1775) & 1 & 1 & $5-10$ & 3 & 1 & & \\
\hline Cydia splendana (HÜBNER, 1799) & & & & & 1 & & \\
\hline Cydia nigricana (FABRICIUS, 1794) & & & & & 3 & & \\
\hline Cydia coniferana (SAXESEN, 1840) & & & & & $5-10$ & & $\mathrm{Pd}$ \\
\hline Cydia pomonella (LINNAEUS, 1758) & & & & & 1 & & \\
\hline Cydia gemmiferana (TREITSCHKE, 1835) & & & 2 & & & & $\mathrm{Pd}$ \\
\hline Cydia funebrana (TREITSCHKE, 1835) & & & & 1 & & & $\mathrm{Pd}$ \\
\hline $\begin{array}{l}\text { Lathronympha strigana (FABRICIUS, } \\
\text { 1775) }\end{array}$ & 1 & & & $5-10$ & 1 & & \\
\hline Pammene fasciana (LINNAEUS, 1761) & & & & & 1 & & $\mathrm{Pd}$ \\
\hline Strophedra weirana (DOUGLAS, 1850) & & 1 & & & & & $\mathrm{Pd}$ \\
\hline $\begin{array}{l}\text { Dichrorampha petiverella (LINNAEUS, } \\
1758 \text { ) }\end{array}$ & & & & & $11-30$ & & \\
\hline $\begin{array}{l}\text { Dichrorampha alpinana (TREITSCHKE, } \\
\text { 1830) }\end{array}$ & & 2 & & & 2 & & $\mathrm{Pd}$ \\
\hline $\begin{array}{l}\text { Dichrorampha plumbagana (TREITSCHKE, } \\
\text { 1830) }\end{array}$ & & & & & $11-30$ & & $\mathrm{Pd}$ \\
\hline $\begin{array}{l}\text { Dichrorampha incognitana (KR.\& } \\
\text { MASLOWSKI, 1933) }\end{array}$ & & & & & 2 & & $\mathrm{Pd}$ \\
\hline $\begin{array}{l}\text { Dichrorampha agilana (TENGSTRÖM, } \\
\text { 1848) }\end{array}$ & & & & & $31-100$ & & $\mathrm{Pd}$ \\
\hline Dichrorampha plumbana (SCOPOLI, 1763) & & 1 & & & & & $\mathrm{Pd}$ \\
\hline \multicolumn{8}{|l|}{$\begin{array}{l}\text { Choreutidae } \\
\end{array}$} \\
\hline Anthophila fabriciana (LINNAEUS, 1767) & & 1 & & & $5-10$ & & \\
\hline $\begin{array}{l}\text { Prochoreutis sehestediana (FABRICIUS, } \\
\text { 1776) }\end{array}$ & & & & & 1 & & $\mathrm{Pd}$ \\
\hline \multicolumn{8}{|l|}{ Epermeniidae } \\
\hline Epermenia falciformis (HAWORTH, 1828) & & 3 & & & & & PL \\
\hline \multicolumn{8}{|l|}{ Pterophoridae } \\
\hline Platyptilia nemoralis (ZELLER, 1841) & & & & & 1 & & $\mathrm{Pd}$ \\
\hline $\begin{array}{l}\text { Platyptilia gonodactyla (DEN. \& SCHIFF., } \\
\text { 1775) }\end{array}$ & 1 & & & & & & \\
\hline $\begin{array}{l}\text { Stenoptilia pterodactyla (LINNAEUS, } \\
\text { 1761) }\end{array}$ & & & & & & 1) & \\
\hline $\begin{array}{l}\text { Stenoptilia bipunctidactyla (SCOPOLI, } \\
1763 \text { ) }\end{array}$ & & & & & 1 & & \\
\hline Geina didactyla (LINNAEUS, 1758) & & 1 & & & & & \\
\hline Oxyptilus pilosellae (ZELLER, 1841) & & & & & & 1) & \\
\hline $\begin{array}{l}\text { Oxyptilus parvidactylus (HAWORTH, } \\
\text { 1811) }\end{array}$ & & & & & $5-10$ & 1) & \\
\hline $\begin{array}{l}\text { Pterophorus pentadactylus (LINNAEUS, } \\
1758 \text { ) }\end{array}$ & $5-10$ & & & & 2 & & \\
\hline Emmelina monodactyla (LINNAEUS, 1758) & & & & & $11-30$ & 1) & \\
\hline \multicolumn{8}{|l|}{ Pyralidae } \\
\hline Pyralis farinalis (LINNAEUS, 1758) & 1 & & & & 1 & & \\
\hline Pyralis regalis (DEN. \& SCHIFF., 1775) & & & & 1 & & & \\
\hline Hypsopygia costalis (FABRICIUS, 1775) & & & & & 1 & & \\
\hline
\end{tabular}




\begin{tabular}{|c|c|c|c|c|c|c|c|}
\hline Cryptoblabes bistriga (HAWORTH, 1811) & & & & & 1 & & \\
\hline Elegia similella (ZINCKEN, 1818) & 1 & & & & 1 & & \\
\hline Ortholepis betulae (GoEZE, 1778) & & & & & 1 & & \\
\hline Pyla fusca (HAWORTH, 1811) & & & & & 1 & & \\
\hline Sciota fumella (EVERSMANN, 1844) & 1 & & & & & & \\
\hline Sciota rhenella (ZINCKEN, 1818) & & & & & 2 & & $\mathrm{Pd}$ \\
\hline Sciota adelphella (FISCHER VON R., 1836) & & & & & 1 & & \\
\hline $\begin{array}{l}\text { Pempelia palumbella (DEN. \& SCHIFF., } \\
\text { 1775) }\end{array}$ & & & & & 2 & & $\mathrm{Pd}$ \\
\hline Pempelia formosa $($ HAWORTH, 1811) & & & 1 & & 2 & & \\
\hline $\begin{array}{l}\text { Dioryctria abietella (DEN. \& SCHIFF., } \\
\text { 1775) }\end{array}$ & & & 1 & 1 & $5-10$ & & \\
\hline Dioryctria simplicella HEINEMANN, 1863 & & & & & 2 & & $\mathrm{Pd}$ \\
\hline $\begin{array}{l}\text { Hypochalcia ahenella (DEN. \& SCHIFF., } \\
\text { 1775) }\end{array}$ & & & & 1 & 1 & & \\
\hline Eurhodope cirrigerella (ZINCKEN, 1818) & & & & & 2 & & \\
\hline Assara terebrella (ZINCKEN, 1818) & 1 & & & 2 & & & \\
\hline $\begin{array}{l}\text { Homoeosoma nimbellum (DUPONCHEL, } \\
\text { 1837) }\end{array}$ & & & & & 1 & 1) & $\operatorname{Pd}(!)$ \\
\hline Ephestia elutella (HÜBNER, 1796) & & & & & 1 & & $\mathrm{Pd}$ \\
\hline Anerastia lotella (HÜBNER, 1813) & 1 & & & & $5-10$ & & \\
\hline Scoparia subfusca HAWORTH, 1811 & & & & & 1 & & $\mathrm{Pd}$ \\
\hline Scoparia ambigualis (TREITSCHKE, 1829) & 1 & 3 & & 1 & $5-10$ & & \\
\hline $\begin{array}{l}\text { Scoparia pyralella (DEN. \& SCHIFF., } \\
1775 \text { ) }\end{array}$ & 1 & & & & $5-10$ & & \\
\hline Eudonia murana (CURTIS, 1827) & 1 & & & & & & $\mathrm{Pd}$ \\
\hline Eudonia truncicolella (STAINTON, 1849) & 1 & & & & & & \\
\hline Chilo phragmitellus (HÜBNER, 1810) & 1 & & & & & & \\
\hline Chrysoteuchia culmella (LINNAEUS, 1758) & 1 & $5-10$ & & & $31-100$ & 1) & \\
\hline Crambus pascuellus (LINNAEUS, 1758) & & & & 2 & $31-100$ & & \\
\hline Crambus ericellus (HÜBNER, 1813) & & & & & 1 & & \\
\hline Crambus pratellus (LINNAEUS, 1758) & 1 & & & & $5-10$ & 1), 2) & \\
\hline Crambus lathoniellus (ZINCKEN, 1817) & $5-10$ & 1 & $5-10$ & $5-10$ & $>100$ & 1), 2) & \\
\hline Catoptria permutatella (HER.-SCH., 1848) & & & & & 2 & & \\
\hline Pediasia luteella (DEN. \& SCHIFF., 1775) & & & & & $5-10$ & 2) & \\
\hline $\begin{array}{l}\text { Platytes cerussellus (DEN. \& SCHIFF., } \\
\text { 1775) }\end{array}$ & $11-30$ & & & & $>100$ & 1), 2) & \\
\hline $\begin{array}{l}\text { Schoenobius gigantellus (DEN. \& SCHIFF., } \\
\text { 1775) }\end{array}$ & 1 & & & & & & \\
\hline Donacaula forficellus (THUNBERG, 1794) & & & & 1 & & & \\
\hline Elophila nymphaeata (LINNAEUS, 1758) & $5-10$ & 1 & & & 1 & & \\
\hline Cataclysta lemnata (LINNAEUS, 1758) & & $5-10$ & & $5-10$ & & & \\
\hline Parapoynx stratiotatum (LINNAEUS, 1758) & 2 & & & & & & \\
\hline Evergestis forficalis (LINNAEUS, 1758) & 1 & & & & & & \\
\hline Evergestis pallidata (HUFNAGEL, 1767) & 1 & & & & & & \\
\hline Evergestis aenealis (DEN. \& SCHIFF., 1775) & & & & & 1 & & \\
\hline Udea lutealis (HÜBNER, 1809) & 1 & & & & & & \\
\hline
\end{tabular}




\begin{tabular}{|c|c|c|c|c|c|c|}
\hline Udea olivalis (DEN. \& SCHIFF., 1775) & 1 & $5-10$ & & 2 & & \\
\hline Udea hamalis (THUNBERG, 1788) & & & & 1 & & \\
\hline Opsibotys fuscalis (DEN. \& SCHIFF., 1775) & & & 2 & & & \\
\hline Loxostege turbidalis (TREITSCHKE, 1829) & 2 & & & & $31-100$ & 1), 2) \\
\hline Pyrausta purpuralis (LINNAEUS, 1758) & 1 & & & & & \\
\hline Pyrausta aerealis (HÜBNER, 1793) & 4 & & & & $31-100$ & \\
\hline Nascia cilialis (HÜBNER, 1796) & 2 & 1 & & & & \\
\hline Sitochroa verticalis (LINNAEUS, 1758) & 1 & & & & $31-100$ & 2) \\
\hline $\begin{array}{l}\text { Perinephela lancealis (DEN. \& SCHIFF., } \\
1775 \text { ) }\end{array}$ & $5-10$ & & & & & \\
\hline Phlyctaenia coronata (HUFNAGEL, 1767) & 1 & & & 1 & 1 & \\
\hline Phlyctaenia perlucidalis (HÜBNER, 1809) & 1 & & & 1 & 1 & \\
\hline Ostrinia palustralis (HÜBNER, 1796) & $11-30$ & & 1 & & 1 & \\
\hline Ostrinia nubilalis (HÜBNER, 1796) & 1 & 1 & & $5-10$ & 1 & \\
\hline Eurrhypara hortulata (LINNAEUS, 1758) & 1 & $11-30$ & & & 1 & \\
\hline Paratalanta hyalinalis (HÜBNER, 1796) & & & & 2 & $11-30$ & \\
\hline Agrotera nemoralis (SCOPOLI, 1763) & 1 & & 1 & & & \\
\hline Diasemia reticularis (LINNAEUS, 1761) & 1 & & & & & \\
\hline \multicolumn{7}{|l|}{ Lasiocampidae } \\
\hline Macrothylacia rubi (LINNAEUS, 1758) & $5-10$ & 1 & & & $5-10$ & 1) \\
\hline Dendrolimus pini (LINNAEUS, 1758) & $11-30$ & & & & $5-10$ & \\
\hline $\begin{array}{l}\text { Gastropacha populifolia (DEN. \& SCHIFF., } \\
1775 \text { ) }\end{array}$ & 1 & & & & 1 & \\
\hline \multicolumn{7}{|l|}{ Sphingidae } \\
\hline Sphinx ligustri LINNAEUS, 1758 & 1 & & & & & \\
\hline Sphinx pinastri LINNAEUS, 1758 & $11-30$ & & 1 & & $5-10$ & \\
\hline Mimas tiliae (LINNAEUS, 1758) & 1 & & & & & \\
\hline Smerinthus ocellatus (LINNAEUS, 1758) & 1 & 1 & 3 & 1 & 1 & \\
\hline Laothoe populi (LINNAEUS, 1758) & 1 & 1 & & 2 & 1 & \\
\hline $\begin{array}{l}\text { Laothoe amurensis (FISCHER V. } \\
\text { WALDHEIM, 1830) }\end{array}$ & 4 & & & & & \\
\hline Deilephila elpenor (LINNAEUS, 1758) & 1 & & & & 1 & \\
\hline Deilephila porcellus (LINNAEUS, 1758) & $5-10$ & & & & & \\
\hline \multicolumn{7}{|l|}{ Hesperiidae } \\
\hline Erynnis tages (LINNAEUS, 1758) & 1 & & & & & \\
\hline Pyrgus malvae (LinNAEUS, 1758) & 1 & & & & & \\
\hline Carterocephalus palaemon (PALLAS, 1771) & 1 & & & & & \\
\hline $\begin{array}{l}\text { Carterocephalus silvicolus (MEIGEN, } \\
\text { 1829) }\end{array}$ & 1 & & & & & \\
\hline Thymelicus sylvestris (PODA, 1761) & $11-30$ & & & 1 & $5-10$ & \\
\hline Hesperia comma (LINNAEUS, 1758) & & & & $5-10$ & & \\
\hline $\begin{array}{l}\text { Ochlodes venatus (BREMER \& GREY, } \\
\text { 1853) }\end{array}$ & 1 & & & 1 & & \\
\hline \multicolumn{7}{|l|}{ Papilionidae } \\
\hline Parnassius mnemosyne (LINNAEUS, 1758) & & & 1 & & & \\
\hline \multicolumn{7}{|l|}{ Pieridae } \\
\hline Pieris brassicae (LINNAEUS, 1758) & $5-10$ & & & & & \\
\hline
\end{tabular}




\begin{tabular}{|c|c|c|c|c|c|c|c|}
\hline Pieris rapae (LINNAEUS, 1758) & 1 & & & & & & \\
\hline Pieris napi (LINNAEUS, 1758) & 1 & & & & & & \\
\hline Pontia daplidice (LINNAEUS, 1758) & 1 & & & & & & \\
\hline $\begin{array}{l}\text { Anthocharis cardamines (LINNAEUS, } \\
\text { 1758) }\end{array}$ & $5-10$ & & & & & & \\
\hline Colias palaeno (LINNAEUS, 1761) & 1 & & & & & & \\
\hline \multicolumn{8}{|l|}{ Lycaenidae } \\
\hline Lycaena phlaeas (LINNAEUS, 1761) & $11-30$ & & & & & & \\
\hline Lycaena dispar (HAWORTH, 1803) & 1 & & & & & & \\
\hline Lycaena hippothoe (LINNAEUS, 1761) & $11-30$ & & & & & & \\
\hline Celastrina argiolus (LINNAEUS, 1758) & 1 & & & & & & \\
\hline Cyaniris semiargus (ROTEMBURG, 1775) & 1 & & & & & & \\
\hline $\begin{array}{l}\text { Polyommatus amandus (SCHNEIDER, } \\
\text { 1792) }\end{array}$ & $5-10$ & & & & 3 & & \\
\hline $\begin{array}{l}\text { Polyommatus icarus (ROTTEMBURG, } \\
\text { 1775) }\end{array}$ & $5-10$ & & & & 1 & & \\
\hline \multicolumn{8}{|l|}{ Nymphalidae } \\
\hline Limenitis populi (LINNAEUS, 1758) & $11-30$ & & & & & & \\
\hline Nymphalis antiopa (LINNAEUS, 1758) & 1 & & & & & & \\
\hline Inachis io (LINNAEUS, 1758) & $5-10$ & & & & & & \\
\hline Aglais urticae (LiNNAEUS, 1758) & $5-10$ & 1 & & 1 & $5-10$ & & \\
\hline Vanessa atalanta (LINNAEUS, 1758) & 1 & & & & & & \\
\hline Polygonia c-album (LINNAEUS, 1758) & 1 & & & & & & \\
\hline Araschnia levana (LINNAEUS, 1758) & 1 & & & & & & \\
\hline Brenthis ino (ROTTEMBURG, 1775) & $5-10$ & $11-30$ & & & $11-30$ & & \\
\hline Boloria selene (DEN. \& SCHIFF., 1775) & 1 & & & & $5-10$ & & \\
\hline Proclossiana eunomia (ESPER, 1799) & $31-100$ & & & & & & \\
\hline Melitaea cinxia (LINNAEUS, 1758) & 1 & & & & & & \\
\hline Melitaea athalia (ROTTEMBURG, 1775) & $11-30$ & & & & $11-30$ & & \\
\hline Euphydryas maturna (LINNAEUS, 1758) & 1 & & & & & & \\
\hline Euphydryas aurinia (ROTTEMBURG, 1775) & 1 & & & & & & \\
\hline \multicolumn{8}{|l|}{\begin{tabular}{|c|} 
Satyridae \\
\end{tabular}} \\
\hline Erebia medusa (DEN. \& SCHIFF., 1775) & 1 & & & & & & \\
\hline Maniola jurtina (LINNAEUS, 1758) & & & & 1 & $5-10$ & & \\
\hline $\begin{array}{l}\text { Coenonympha pamphilus (LINNAEUS, } \\
\text { 1758) }\end{array}$ & $11-30$ & & & & $5-10$ & 1) & \\
\hline Coenonympha hero (LINNAEUS, 1761) & & & 2 & & & & \\
\hline $\begin{array}{l}\text { Coenonympha glycerion (BORKHAUSEN, } \\
\text { 1788) }\end{array}$ & $11-30$ & & & & $11-30$ & 1) & \\
\hline Pararge aegeria (LINNAEUS, 1758) & 1 & & & & & & \\
\hline Lasiommata megera (LINNAEUS, 1767) & 1 & & & & & & \\
\hline Lasiommata maera (LINNAEUS, 1758) & & & & & & 3) & \\
\hline Lopinga achine (SCOPOLI, 1763) & & & & & & 3) & \\
\hline \multicolumn{8}{|l|}{ Drepanidae } \\
\hline Falcaria lacertinaria (LINNAEUS, 1758) & 1 & & 2 & & 1 & & \\
\hline Drepana falcataria (LINNAEUS, 1758) & 1 & 2 & 3 & 1 & 1 & & \\
\hline Drepana curvatula (BORKHAUSEN, 1790) & $5-10$ & & & 1 & 2 & & \\
\hline
\end{tabular}




\begin{tabular}{|c|c|c|c|c|c|c|c|}
\hline Sabra harpagula (ESPER, 1786) & 1 & & & & & & \\
\hline Thyatira batis (LINNAEUS, 1758) & 1 & & & 1 & 1 & & \\
\hline Habrosyne pyritoides (HUFNAGEL, 1766) & $5-10$ & 1 & & & 1 & & \\
\hline Tethea or (DEN. \& SCHIFF., 1775) & 1 & & 1 & & & & \\
\hline Tetheella fluctuosa (HÜBNER, 1803) & & & & 1 & 1 & & \\
\hline Ochropacha duplaris (LINNAEUS, 1761) & $5-10$ & & & & & & \\
\hline \multicolumn{8}{|l|}{ Geometridae } \\
\hline Abraxas sylvatus (SCOPOLI, 1763) & & 2 & & & 1 & & \\
\hline Lomaspilis marginata (LINNAEUS, 1758) & $11-30$ & $5-10$ & 1 & $11-30$ & $5-10$ & & \\
\hline Lomaspilis opis (BUTLER, 1878) & 13 & & & 3 & 4 & & \\
\hline Ligdia adustata (DEN. \& SCHIFF., 1775) & $5-10$ & 2 & & & $5-10$ & & \\
\hline Chiasmia notata (LINNAEUS, 1758) & 1 & 2 & $5-10$ & 1 & $5-10$ & & \\
\hline $\begin{array}{l}\text { Chiasmia alternata (DEN. \& SCHIFF., } \\
1775 \text { ) }\end{array}$ & 1 & 1 & & 1 & $5-10$ & & \\
\hline Chiasmia signaria (HÜBNER, 1809) & 1 & & & 1 & 1 & 2) & \\
\hline Chiasmia liturata (CLERCK, 1759) & & & $5-10$ & 3 & $5-10$ & 1), 2) & \\
\hline Chiasmia wauaria (LINNAEUs, 1758) & 1 & 2 & & & & & \\
\hline Chiasmia clathrata (LINNAEUS, 1758) & $5-10$ & & $5-10$ & 1 & $>100$ & 1) & \\
\hline Chiasmia brunneata (THUNBERG, 1784) & & & & & 2 & & \\
\hline $\begin{array}{l}\text { Pseudopanthera macularia (LINNAEUS, } \\
1758 \text { ) }\end{array}$ & 1 & & & & & & \\
\hline Plagodis pulveraria (LINNAEUS, 1758) & 1 & & 1 & 1 & & & \\
\hline Plagodis dolabraria (LINNAEUS, 1767) & 1 & & & & 1 & & \\
\hline Petrophora chlorosata (SCOPOLI, 1763) & 1 & & $5-10$ & & $5-10$ & & \\
\hline $\begin{array}{l}\text { Opisthograptis luteolata (LINNAEUS, } \\
1758 \text { ) }\end{array}$ & & & & & 1 & & \\
\hline Cepphis advenaria (HÜBNER, 1790) & $11-30$ & $5-10$ & $11-30$ & $5-10$ & $11-30$ & & \\
\hline Odontopera bidentata (CLERCK, 1759) & $5-10$ & & 1 & & & & \\
\hline Angerona prunaria (LINNAEUS, 1758) & 1 & 1 & & 2 & 2 & & \\
\hline Lycia hirtaria $($ CLERCK, 1759) & 1 & & & & & & \\
\hline Biston betularius (LINNAEUS, 1758) & 1 & 1 & & & $5-10$ & & \\
\hline Alcis repandata (LINNAEUS, 1758) & & 1 & & 1 & 1 & & \\
\hline $\begin{array}{l}\text { Hypomecis roboraria (DEN. \& SCHIFF., } \\
1775 \text { ) }\end{array}$ & 1 & 2 & & 2 & 1 & & \\
\hline Hypomecis punctinalis (SCOPOLI, 1763) & 1 & 1 & 1 & 1 & $5-10$ & & \\
\hline Parectropis similaria (HuFNAGEL, 1767) & 1 & & & 1 & & & \\
\hline $\begin{array}{l}\text { Aethalura punctulata (DEN. \& SCHIFF., } \\
\text { 1775) }\end{array}$ & & 1 & $5-10$ & & & & \\
\hline Ematurga atomaria (LINNAEUS, 1758) & $5-10$ & & & 1 & $5-10$ & 2) & \\
\hline Bupalus piniarius (LINNAEUS, 1758) & & & $5-10$ & & $>100$ & & \\
\hline Cabera pusaria (LINNAEUS, 1758) & 1 & 1 & $5-10$ & 2 & 1 & & \\
\hline Cabera exanthemata (SCOPOLI, 1763) & $5-10$ & 2 & $5-10$ & $11-30$ & 4 & & \\
\hline $\begin{array}{l}\text { Lomographa bimaculata (FABRICIUS, } \\
1775 \text { ) }\end{array}$ & 1 & 1 & & & 1 & & \\
\hline $\begin{array}{l}\text { Lomographa temerata (DEN. \& SCHIFF., } \\
\text { 1775) }\end{array}$ & 1 & & & & 2 & & \\
\hline Campaea margaritata (LINNAEUS, 1767) & & & & 1 & 1 & & $\operatorname{Pd}(!)$ \\
\hline
\end{tabular}




\begin{tabular}{|c|c|c|c|c|c|c|c|}
\hline Hylaea fasciaria (LINNAEUS, 1758) & & & & & 2 & & \\
\hline Charissa ambiguata (DUPONCHEL, 1830) & & & & & 1 & & $\operatorname{Pd}(!)$ \\
\hline Siona lineata $(\mathrm{SCOPOLI}, 1763)$ & & & & 1 & $31-100$ & & $\operatorname{Pd}(!)$ \\
\hline Geometra papilionaria (LINNAEUS, 1758) & & 1 & & 1 & $5-10$ & & \\
\hline $\begin{array}{l}\text { Comibaena bajularia (DEN. \& SCHIFF., } \\
1775 \text { ) }\end{array}$ & 1 & & & & $5-10$ & & \\
\hline $\begin{array}{l}\text { Antonechloris smaragdaria (FABRICIUS, } \\
\text { 1787) }\end{array}$ & 1 & & & & $11-30$ & & \\
\hline Chlorissa viridata (LINNAEUS, 1758) & & & & & 2 & & \\
\hline Jodis putata (LINNAEUS, 1758) & 1 & & & 1 & 1 & & \\
\hline Cyclophora pendularia (CLERCK, 1759) & & & 1 & 2 & & & \\
\hline Cyclophora annularia (FABRICIUS, 1775) & 1 & & & & & & \\
\hline Cyclophora albipunctata (HUFNAGEL, 1767) & 1 & & 1 & 1 & 1 & & \\
\hline Cyclophora porata (LINNAEUS, 1767) & & & & & 2 & & \\
\hline Cyclophora punctaria (LINNAEUS, 1758) & 1 & & $5-10$ & 1 & $11-30$ & & \\
\hline Cyclophora linearia (HÜBNER, 1799) & & & 1 & & 1 & & \\
\hline Timandra comae SCHMIDT, 1931 & $11-30$ & 1 & $5-10$ & 1 & $5-10$ & & \\
\hline Scopula immorata (LINNAEUS, 1758) & $5-10$ & & & $5-10$ & $5-10$ & & \\
\hline Scopula nemoraria (HÜBNER, 1799) & & & $5-10$ & & & & \\
\hline Scopula nigropunctata (HUFNAGEL, 1767) & & & & 1 & $5-10$ & & \\
\hline Scopula ornata (SCOPOLI, 1763) & 1 & & & & & & \\
\hline Scopula rubiginata (HUFNAGEL, 1767) & 1 & & & & $5-10$ & & \\
\hline Scopula immutata (LINNAEUS, 1758) & $5-10$ & & & $5-10$ & & & \\
\hline Scopula ternata SCHRANK, 1802 & $5-10$ & & & $5-10$ & $5-10$ & 2) & \\
\hline Scopula floslactata (HAWORTH, 1809) & 1 & & & & & & \\
\hline Idaea dilutaria (HÜBNER, 1799) & & & 1 & & & & $\mathrm{Pd}$ \\
\hline Idaea aversata (LINNAEUS, 1758) & & & & & 1 & & \\
\hline Lythria cruentaria (HUFNAGEL, 1767) & & & & & $5-10$ & 2) & \\
\hline Orthonama vittata (BORKHAUSEN, 1794) & $5-10$ & 1 & $11-30$ & 4 & 1 & & \\
\hline $\begin{array}{l}\text { Xanthorhoe biriviata (BORKHAUSEN, } \\
\text { 1794) }\end{array}$ & 1 & & & & & & \\
\hline Xanthorhoe designata (HuFNAGEL, 1767) & 1 & & & 1 & & & \\
\hline $\begin{array}{l}\text { Xanthorhoe spadicearia (DEN. \& SCHIFF., } \\
1775 \text { ) }\end{array}$ & 1 & & $5-10$ & 1 & & & \\
\hline Xanthorhoe ferrugata (CLERCK, 1759) & 1 & 2 & & & 3 & & \\
\hline $\begin{array}{l}\text { Xanthorhoe quadrifasciata (CLERCK, } \\
\text { 1759) }\end{array}$ & & & & & 1 & & \\
\hline $\begin{array}{l}\text { Xanthorhoe montanata (DEN. \& SCHIFF., } \\
1775 \text { ) }\end{array}$ & 1 & 1 & & $11-30$ & & & \\
\hline Xanthorhoe fluctuata (LINNAEUS, 1758) & & & & & 1 & & \\
\hline Catarhoe cuculata (HUFNAGEL, 1767) & & & 1 & & 2 & & \\
\hline Epirrhoe tristata (LINNAEUS, 1758) & 1 & & & 1 & $5-10$ & & \\
\hline Epirrhoe alternata (MÜLLER, 1764) & 1 & $5-10$ & $5-10$ & 1 & $11-30$ & 1) & \\
\hline Epirrhoe rivata (HÜBNER, 1813) & & & & 1 & & & \\
\hline Epirrhoe tartuensis MOELS, 1965 & & 2 & 2 & & & & \\
\hline $\begin{array}{l}\text { Costaconvexa polygrammata } \\
\text { (BORKHAUSEN, 1794) }\end{array}$ & & & & & 1 & & \\
\hline
\end{tabular}




\begin{tabular}{|c|c|c|c|c|c|c|}
\hline $\begin{array}{l}\text { Camptogramma bilineatum (LINNAEUS, } \\
1758 \text { ) }\end{array}$ & 1 & & & & $5-10$ & \\
\hline Mesoleuca albicillata (LINNAEUS, 1758) & 1 & & & & 1 & \\
\hline $\begin{array}{l}\text { Lampropteryx suffumata (DEN. \& SCHIFF., } \\
\text { 1775) }\end{array}$ & 1 & & & & & \\
\hline $\begin{array}{l}\text { Lampropteryx otregiata (METCALFE, } \\
\text { 1917) }\end{array}$ & 2 & & & & & \\
\hline Cosmorhoe ocellata (LINNAEUS, 1758) & 1 & & $5-10$ & 1 & $5-10$ & \\
\hline Eulithis prunata (LINNAEUS, 1758) & 1 & 1 & & & & \\
\hline Eulithis populata (LINNAEUS, 1758) & & & & 1 & 1 & \\
\hline Eulithis mellinata (FABRICIUS, 1787) & $5-10$ & 2 & & & 2 & \\
\hline Eulithis pyraliata (DEN. \& SCHIFF., 1775) & 1 & & & & & \\
\hline $\begin{array}{l}\text { Ecliptopera silaceata (DEN. \& SCHIFF., } \\
1775 \text { ) }\end{array}$ & & & & 1 & & \\
\hline Ecliptopera capitata (HER.-SCH., 1839) & 1 & & 1 & & 1 & \\
\hline Chloroclysta siterata (HUFNAGEL, 1767) & & & 1 & & & \\
\hline Chloroclysta truncata (HUFNAGEL, 1767) & 1 & 2 & & $5-10$ & 1 & \\
\hline Thera obeliscata (HÜBNER, 1787) & 1 & & $11-30$ & $5-10$ & $5-10$ & \\
\hline Thera variata (DEN. \& SCHIFF., 1775) & 1 & & & 1 & & \\
\hline $\begin{array}{l}\text { Eustroma reticulatum (DEN. \& SCHIFF., } \\
1775 \text { ) }\end{array}$ & 1 & 1 & & & & \\
\hline Electrophaes corylata (THUNBERG, 1792) & 1 & & & & 1 & \\
\hline Colostygia pectinataria $(\mathrm{KNOCH}, 1781)$ & $5-10$ & & & 1 & 1 & \\
\hline Hydriomena furcata (THUNBERG, 1784) & & & $5-10$ & & & \\
\hline $\begin{array}{l}\text { Hydriomena impluviata (DEN. \& SCHIFF., } \\
\text { 1775) }\end{array}$ & $5-10$ & $5-10$ & $5-10$ & 1 & 1 & \\
\hline Horisme tersata (DEN. \& SCHIFF., 1775) & & & & & 1 & \\
\hline Rheumaptera undulata (LINNAEUS, 1758) & $11-30$ & & $11-30$ & 1 & 1 & \\
\hline Euphyia biangulata (HAWORTH, 1809) & 2 & & & & & \\
\hline Euphyia unangulata (HAWORTH, 1809) & 1 & 1 & & 1 & 1 & \\
\hline $\begin{array}{l}\text { Perizoma alchemillatum (LINNAEUS, } \\
1758 \text { ) }\end{array}$ & & 1 & & 1 & $11-30$ & \\
\hline $\begin{array}{l}\text { Perizoma albulatum (DEN. \& SCHIFF., } \\
\text { 1775) }\end{array}$ & 1 & & & & & \\
\hline $\begin{array}{l}\text { Perizoma flavofasciatum (THUNBERG, } \\
\text { 1792) }\end{array}$ & 1 & & & & & \\
\hline Perizoma didymatum (LINNAEUS, 1758) & 1 & & & & & \\
\hline Eupithecia abietaria (GOEZE, 1781) & $5-10$ & & & 1 & $5-10$ & \\
\hline Eupithecia venosata (FABRICIUS, 1787) & & & & & 1 & \\
\hline Eupithecia tripunctaria HER.-SCH., 1852 & & & 1 & & & \\
\hline Eupithecia tantillaria BOISDUVAL, 1840 & $11-30$ & & $31-100$ & & 1 & \\
\hline Eupithecia selinata HER.-SCH., 1861 & & & & 3 & & \\
\hline $\begin{array}{l}\text { Eupithecia plumbeolata (HAWORTH, } \\
\text { 1809) }\end{array}$ & $5-10$ & & $5-10$ & $5-10$ & $5-10$ & \\
\hline $\begin{array}{l}\text { Eupithecia centaureata (DEN. \& SCHIFF., } \\
\text { 1775) }\end{array}$ & & & & & 2 & \\
\hline Eupithecia vulgata (HAWORTH, 1809) & & & $5-10$ & & & \\
\hline
\end{tabular}




\begin{tabular}{|c|c|c|c|c|c|c|c|}
\hline Eupithecia assimilata DOUBLEDAY, 1856 & & & & 1 & 2 & & \\
\hline Eupithecia satyrata (HÜBNER, 1813) & $5-10$ & & $11-30$ & & & & \\
\hline Eupithecia intricata (ZETTERSTEDT, 1839) & & & $31-100$ & 1 & & & \\
\hline Eupithecia absinthiata (CLERCK, 1759) & & & & & 1 & & \\
\hline $\begin{array}{l}\text { Eupithecia subumbrata (DEN. \& SCHIFF., } \\
\text { 1775) }\end{array}$ & 1 & & & 1 & 1 & 1) & \\
\hline Eupithecia subfuscata (HAWORTH, 1809) & 1 & & 1 & & 1 & & \\
\hline Eupithecia exiguata (HÜBNER, 1813) & $5-10$ & & $11-30$ & 1 & & & \\
\hline $\begin{array}{l}\text { Eupithecia succenturiata (LINNAEUS, } \\
1758 \text { ) }\end{array}$ & & & & & 1 & & \\
\hline Rhinoprora debiliata (HÜBNER, 1817) & & 1 & & 1 & 1 & & \\
\hline Anticollix sparsata (TREITSCHKE, 1828) & 1 & & 1 & & & & \\
\hline Hydrelia flammeolaria (HUFNAGEL, 1767) & 1 & $5-10$ & $5-10$ & $5-10$ & 1 & & \\
\hline Hydrelia sylvata (DEN. \& SCHIFF., 1775) & & 1 & & & & & \\
\hline Euchoeca nebulata (SCOPOLI, 1763) & 1 & 1 & $5-10$ & $5-10$ & 1 & & \\
\hline Asthena albulata (HUFNAGEL, 1767) & 1 & & & & & & \\
\hline Pterapherapteryx sexalata (RETZIUS, 1783) & 1 & & & 4 & $5-10$ & & \\
\hline \multicolumn{8}{|l|}{ Notodontidae } \\
\hline Clostera curtula (LINNAEUS, 1758) & 1 & & 1 & & & & \\
\hline Clostera pigra (HUFNAGEL, 1766) & 1 & & $5-10$ & & 1 & & \\
\hline Cerura erminea (ESPER, 1783) & & & & & 1 & & \\
\hline Furcula bicuspis (BORKHAUSEN, 1790) & 1 & & & & & & \\
\hline Notodonta dromedarius (LINNAEUS, 1767) & 1 & & & $5-10$ & 1 & & \\
\hline Notodonta ziczac (LINNAEUS, 1758) & 1 & & 1 & & 1 & & \\
\hline $\begin{array}{l}\text { Drymonia dodonaea (DEN. \& SCHIFF., } \\
\text { 1775) }\end{array}$ & $5-10$ & & & $5-10$ & $5-10$ & & \\
\hline Drymonia velitaris (HUFNAGEL, 1766) & 1 & & & & 3 & & $\mathrm{Pd}$ \\
\hline Drymonia obliterata (ESPER, 1785) & & 2 & & & & & \\
\hline Pheosia gnoma (FABRICIUS, 1776) & 1 & & $5-10$ & & 1 & & \\
\hline Pterostoma palpinum (CLERCK, 1759) & & & & 1 & & & \\
\hline $\begin{array}{l}\text { Leucodonta bicoloria (DEN. \& SCHIFF., } \\
1775 \text { ) }\end{array}$ & 1 & & & 3 & 1 & & \\
\hline Ptilodon capucina (LINNAEUS, 1758) & 1 & 1 & 1 & $5-10$ & 1 & & \\
\hline Gluphisia crenata (ESPER, 1785) & 3 & 2 & & & 3 & & \\
\hline Phalera bucephala (LinNAEUS, 1758) & $5-10$ & 2 & & $5-10$ & 1 & & \\
\hline Stauropus fagi (LINNAEUS, 1758) & 1 & & & & 1 & & \\
\hline Harpyia milhauseri (FABRICIUS, 1775) & & & 1 & & & & \\
\hline \multicolumn{8}{|l|}{ Noctuidae } \\
\hline Moma alpium (OSBECK, 1778) & 1 & & & & 2 & & \\
\hline $\begin{array}{l}\text { Acronicta megacephala (DEN. \& SCHIFF., } \\
\text { 1775) }\end{array}$ & & 1 & & 1 & $5-10$ & & \\
\hline Acronicta cuspis (HÜBNER, 1813) & 1 & & & & 2 & & \\
\hline Acronicta leporina (LINNAEUS, 1758) & 1 & 3 & & 1 & 1 & & \\
\hline $\begin{array}{l}\text { Acronicta strigosa (DEN. \& SCHIFF., } \\
1775 \text { ) }\end{array}$ & 2 & 1 & & & 1 & & \\
\hline $\begin{array}{l}\text { Acronicta auricoma (DEN. \& SCHIFF., } \\
\text { 1775) }\end{array}$ & & & $5-10$ & & & & \\
\hline
\end{tabular}




\begin{tabular}{|c|c|c|c|c|c|c|}
\hline Acronicta rumicis (LINNAEUS, 1758) & 1 & 1 & & & 1 & \\
\hline $\begin{array}{l}\text { Craniophora ligustri (DEN. \& SCHIFF., } \\
\text { 1775) }\end{array}$ & 1 & & & & & \\
\hline Simyra albovenosa (GOEZE, 1781) & & & 1 & & & \\
\hline Macrochilo cribrumalis (HÜBNER, 1793) & & & & 1 & & \\
\hline Herminia tarsicrinalis $(\mathrm{KNOCH}, 1782)$ & 1 & 1 & & 1 & $5-10$ & \\
\hline $\begin{array}{l}\text { Herminia grisealis (DEN. \& SCHIFF., } \\
1775 \text { ) }\end{array}$ & 1 & & & 1 & & \\
\hline $\begin{array}{l}\text { Polypogon tentacularius (LINNAEUS, } \\
1758 \text { ) }\end{array}$ & 1 & 1 & & & & \\
\hline Pechipogo strigilata (LINNAEUS, 1758) & 1 & & $5-10$ & & 1 & \\
\hline Schrankia taenialis (HÜBNER, 1809) & 1 & $5-10$ & & 4 & 4 & $\mathrm{Pd}$ \\
\hline Schrankia costaestrigalis (STEPHENS, 1834) & & & & 3 & 1 & \\
\hline Hypenodes humidalis DOUBLEDAY, 1850 & & & & 1 & & \\
\hline Lygephila viciae (HÜBNER, 1822) & 1 & & & & $5-10$ & \\
\hline Callistege mi (CLERCK, 1759) & 1 & & & & & \\
\hline Euclidia glyphica (LINNAEUS, 1758) & 1 & & & & 1 & \\
\hline Laspeyria flexula (DEN. \& SCHIFF., 1775) & 1 & & & & $5-10$ & \\
\hline Hypena crassalis (FABRICIUS, 1787) & & & & & $5-10$ & \\
\hline Hypena rostralis (LINNAEUS, 1758) & & & $5-10$ & & & \\
\hline Hypena proboscidalis (LINNAEUS, 1758) & & $5-10$ & & & $11-30$ & \\
\hline Rivula sericealis (SCOPOLI, 1763) & $11-30$ & $11-30$ & $5-10$ & $5-10$ & $11-30$ & \\
\hline $\begin{array}{l}\text { Colobochyla salicalis (DEN. \& SCHIFF., } \\
1775 \text { ) }\end{array}$ & 1 & 2 & & 1 & $5-10$ & \\
\hline Plusia festucae (LINNAEUS, 1758) & & & & & 1 & \\
\hline Plusia putnami (GROTE, 1873) & 1 & & & & & \\
\hline Diachrysia chrysitis (LINNAEUS, 1758) & 1 & 2 & & 1 & 1 & \\
\hline Autographa gamma (LINNAEUS, 1758) & & & 1 & & & \\
\hline Autographa pulchrina (HAWORTH, 1809) & 1 & 4 & & & 1 & \\
\hline Autographa jota (LINNAEUS, 1758) & 1 & 1 & & 2 & & \\
\hline Abrostola tripartita (HUFNAGEL, 1766) & 1 & 1 & & & & \\
\hline Abrostola triplasia (LINNAEUS, 1758) & 1 & & & 1 & & \\
\hline Emmelia trabealis (SCOPOLI, 1763) & & & & & 2 & \\
\hline Protodeltote pygarga (HUFNAGEL, 1766) & $5-10$ & & & $5-10$ & 1 & \\
\hline Deltote bankiana (FABRICIUS, 1775) & $11-30$ & & & $11-30$ & 1 & \\
\hline Deltote uncula (CLERCK, 1759) & $5-10$ & & & 1 & & \\
\hline Deltote deceptoria $(\mathrm{SCOPOLI}, 1763)$ & 1 & & & & & \\
\hline $\begin{array}{l}\text { Pseudeustrotia candidula (DEN. \& } \\
\text { SCHIFF., 1775) }\end{array}$ & $5-10$ & & & & 1 & \\
\hline $\begin{array}{l}\text { Trisateles emortualis (DEN. \& SCHIFF., } \\
1775 \text { ) }\end{array}$ & 1 & & & & & \\
\hline Cucullia umbratica (LINNAEUS, 1758) & 1 & & & 1 & & \\
\hline Elaphria venustula (HÜBNER, 1790) & 1 & 1 & $5-10$ & 1 & $>100$ & \\
\hline Caradrina morpheus (HUFNAGEL, 1766) & & & & 1 & & \\
\hline Hoplodrina octogenaria (GOEZE, 1781) & & & & & 2 & \\
\hline $\begin{array}{l}\text { Hoplodrina ambigua (DEN. \& SCHIFF., } \\
\text { 1775) }\end{array}$ & & & & 1 & & \\
\hline
\end{tabular}




\begin{tabular}{|c|c|c|c|c|c|c|c|}
\hline $\begin{array}{l}\text { Charanyca trigrammica (HUFNAGEL, } \\
1766 \text { ) }\end{array}$ & $5-10$ & & & & $5-10$ & & \\
\hline $\begin{array}{l}\text { Dypterygia scabriuscula (LINNAEUS, } \\
1758 \text { ) }\end{array}$ & 1 & 1 & 1 & 3 & 1 & & \\
\hline Rusina ferruginea (ESPER, 1785) & $11-30$ & 1 & & 1 & 2 & & \\
\hline Trachea atriplicis (LINNAEUS, 1758) & 1 & & & & & & \\
\hline Euplexia lucipara (LINNAEUS, 1758) & 1 & 1 & & 1 & 1 & & \\
\hline Hyppa rectilinea (ESPER, 1788) & 1 & & & & 1 & & \\
\hline Actinotia polyodon (CLERCK, 1759) & & & & & 1 & & \\
\hline Eucarta virgo (TREITSCHKE, 1835) & 1 & & & & 1 & & $\mathrm{Pd}$ \\
\hline Apamea sublustris (ESPER, 1788) & 1 & & & & $5-10$ & & \\
\hline Apamea crenata (HUFNAGEL, 1766) & 1 & 1 & & 3 & & & \\
\hline Apamea remissa (HÜBNER, 1809) & & & & 1 & 1 & & \\
\hline Apamea unanimis (HÜBNER, 1813) & 1 & 1 & & 1 & & & \\
\hline Apamea anceps (DEN. \& SCHIFF., 1775) & & & & & 1 & & \\
\hline Apamea sordens (HUFNAGEL, 1766) & 1 & & & 3 & & 1) & \\
\hline Oligia strigilis (LINNAEUS, 1758) & & 1 & & & $5-10$ & & \\
\hline Oligia latruncula (DEN. \& SCHIFF., 1775) & 1 & & & 1 & 1 & & \\
\hline Oligia fasciuncula (HAWORTH, 1809) & & & & & 1 & & \\
\hline Discestra trifolii (HUFNAGEL, 1766) & 1 & & & & & & \\
\hline Lacanobia w-latinum (HUFNAGEL, 1766) & & & 1 & & 1 & & \\
\hline Lacanobia splendens (HÜBNER, 1808) & 1 & & 1 & 1 & & & \\
\hline Lacanobia oleracea (LINNAEUS, 1758 ) & 1 & & & & 1 & & \\
\hline $\begin{array}{l}\text { Lacanobia contigua (DEN. \& SCHIFF., } \\
1775 \text { ) }\end{array}$ & & & & & $5-10$ & & \\
\hline Lacanobia thalassina (HUFNAGEL, 1766) & 1 & & 1 & & 1 & & \\
\hline Lacanobia suasa (DEN. \& SCHIFF., 1775) & & & 1 & 2 & 1 & & \\
\hline Hada plebeja (LinNAEUS, 1761) & 1 & & $5-10$ & $5-10$ & $5-10$ & & \\
\hline Hadena bicruris (HuFNAGEL, 1766) & & & & & 1 & & \\
\hline Hadena compta (DEN. \& SCHIFF., 1775) & 1 & & & & & & \\
\hline Hadena confusa (HUFNAGEL, 1766) & 1 & & & & 1 & & \\
\hline Hadena luteago (DEN. \& SCHIFF., 1775) & & & & & 1 & & \\
\hline Hadena rivularis (FABRICIUS, 1775) & 1 & 1 & 1 & 1 & 1 & & \\
\hline Heliophobus reticulatus (GoEZE, 1781) & & & & & 1 & 1) & \\
\hline Melanchra pisi (LINNAEUS, 1758) & $5-10$ & & & 1 & $5-10$ & & \\
\hline Polia bombycina (HUFNAGEL, 1766) & & & & & $5-10$ & & \\
\hline Polia hepatica (CLERCK, 1759) & & & & & 2 & 1) & \\
\hline Polia nebulosa (HuFNAGEL, 1766) & 1 & & & & 1 & & \\
\hline Mythimna turca (LINNAEUS, 1761) & 1 & & & & 1 & & \\
\hline $\begin{array}{l}\text { Mythimna conigera (DEN. \& SCHIFF., } \\
\text { 1775) }\end{array}$ & & & & & 1 & & \\
\hline $\begin{array}{l}\text { Mythimna albipuncta (DEN. \& SCHIFF., } \\
1775 \text { ) }\end{array}$ & & & & & 1 & & \\
\hline Mythimna pallens (LINNAEUS, 1758) & 1 & & & & 1 & & \\
\hline Mythimna obsoleta (HÜBNER, 1803) & 1 & 1 & & & & & \\
\hline Mythimna comma (LINNAEUS, 1761) & $5-10$ & & & & $5-10$ & 1) & \\
\hline Mythimna l-album (LINNAEUS, 1767) & & & & 1 & & & \\
\hline
\end{tabular}




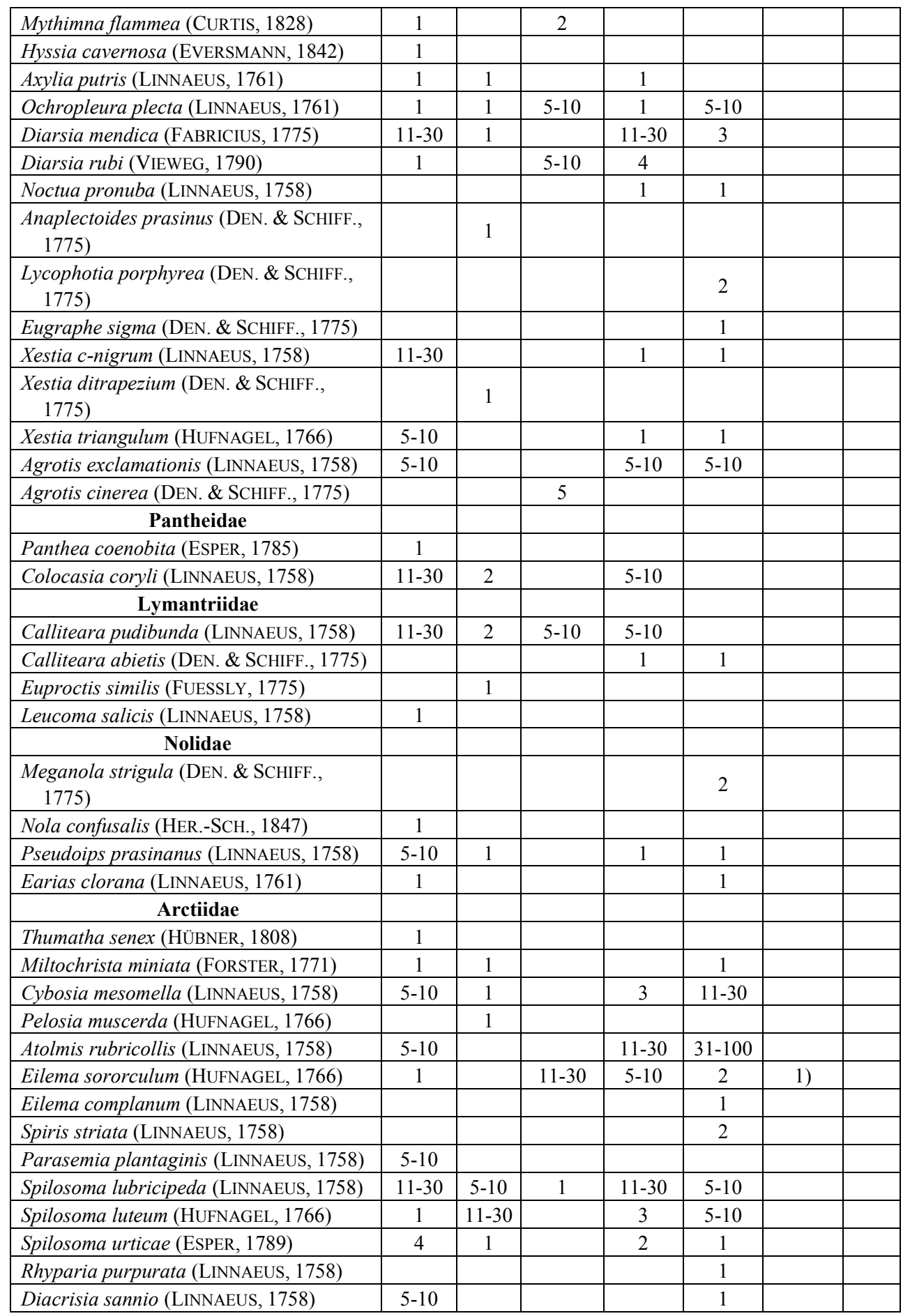




\begin{tabular}{|l|l|l|l|l|l|l|l|}
\hline Callimorpha dominula (LINNAEUS, 1758) & 1 & & & & 1 & & \\
\hline Tyria jacobaeae (LINNAEUS, 1758) & & 1 & & & & & \\
\hline
\end{tabular}

Received: January 13, 2011

Accepted: February 25, 2011 